Journal of the Society for the Study of Architecture in Canada Le Journal de la Société pour l'étude de l'architecture au Canada

\title{
Repenser la fabrique des espaces publics de la ville contemporaine
}

\section{L’exemple du prototype du Virage à Montréal}

Jonathan Cha

Volume 45, numéro 1, 2020

URI : https://id.erudit.org/iderudit/1075074ar

DOI : https://doi.org/10.7202/1075074ar

Aller au sommaire du numéro

Éditeur(s)

SSAC-SEAC

ISSN

2563-8696 (numérique)

Découvrir la revue

Citer cet article

Cha, J. (2020). Repenser la fabrique des espaces publics de la ville contemporaine : l'exemple du prototype du Virage à Montréal. Journal of the Society for the Study of Architecture in Canada / Le Journal de la Société pour l'étude de l'architecture au Canada, 45(1), 23-41.

https://doi.org/10.7202/1075074ar d'utilisation que vous pouvez consulter en ligne. 


\section{REPENSER LA FABRIQUE DES ESPACES PUBLICS DE LA VILLE CONTEMPORAINE \\ L'exemple du prototype du Virage à Montréal}

JONATHAN CHA est docteur en aménagement de l'espace et urbanisme, urbanologue, architecte paysagiste membre de l'Association des architectes paysagistes du Québec (AAPQ) et du Canada (AAPC), Next City Vanguard Alumni, consultant en patrimoine et conseiller spécial en aménagement à la direction générale du parc Jean-Drapeau à Montréal.

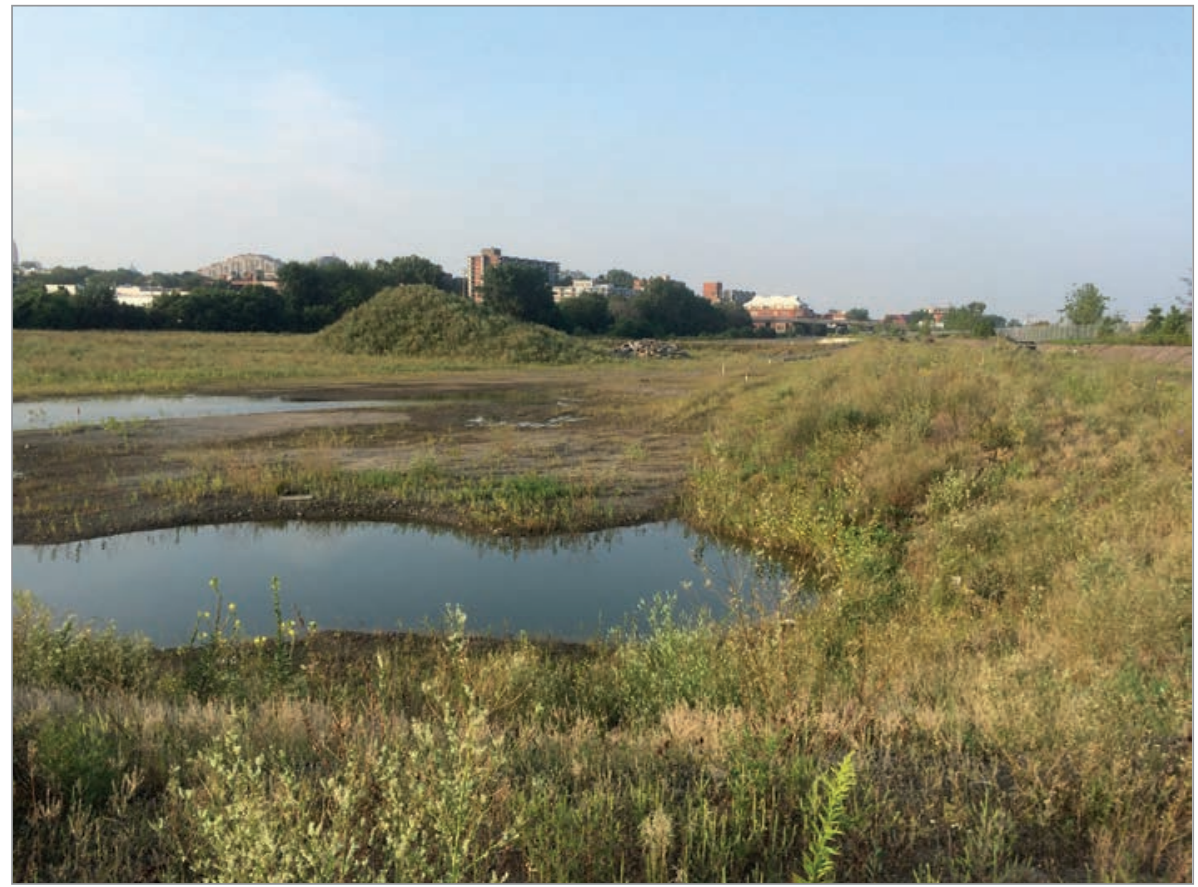

ILL. 1. LA FRICHE DE L'ANCIENNE COUR DE TRIAGE DU CANADIEN PACIFIQUE AVANT LE DÉBUT DU PROJET. | CAMPUS MLL, UNIVERSTÉ DE MONTREAL.

\section{$>$ JONATHAN CHA}

\author{
MISE EN CONTEXTE
}

'article propose une approche différente pour alimenter la fabrique des espaces publics de la ville contemporaine à partir de l'expérience d'une rechercheaction réalisée sur une ancienne cour de triage en requalification : celle-ci accueillera un quartier intégré développé autour du nouveau Campus MIL de I'Université de Montréal. En occupant le rôle de codirecteur du Virage (un espace extérieur formé d'un assemblage de conteneurs recyclés et entourés de jardins potagers nommé projets éphémères), l'auteur a misé sur l'expérimentation pour questionner le devenir des espaces publics, tant dans leur processus constructif que dans leur rôle dans la ville. La réflexion s'est effectuée en exploitant le potentiel du lieu, en testant des processus et des contenus et en misant sur les pratiques spatiales des citoyens. Le prototype tourné vers l'action et la créativité est ancré dans le territoire et porte un intérêt à l'intégration de la communauté locale. Inscrit dans le mouvement de l'urbanisme tactique et des communs, il propose un nouveau modèle de fabrication et d'occupation des espaces publics complémentaire aux typologies héritées de la ville classique et répondant aux enjeux de la ville contemporaine.

\section{LE VIRAGE}

Le Virage est le nom d'un espace extérieur formé d'un assemblage de conteneurs recyclés entouré de jardins potagers (projets éphémères) implanté pour une durée limitée ( 5 ans) et destiné à recevoir des activités éducatives, publiques et 


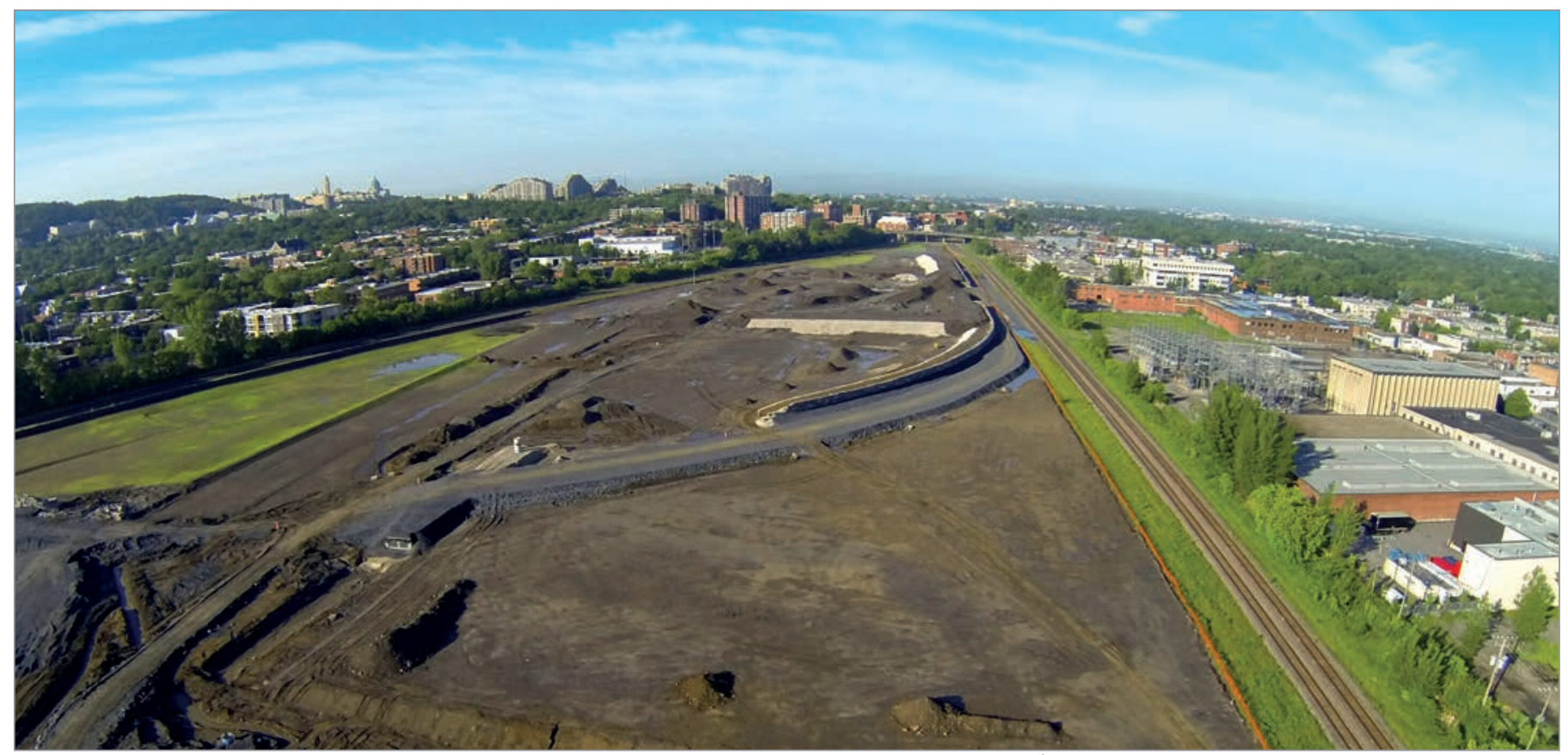

ILL. 2. LE NIVELLEMENT DE L'ANCIENNE COUR DE TRIAGE DU CANADIEN PACIFIQUE ET LE REPOSITIONNEMENT DES VOIES FERRÉES. | CAMPUS MIL, UNIVERSITÉ DE MONTRÉAL.

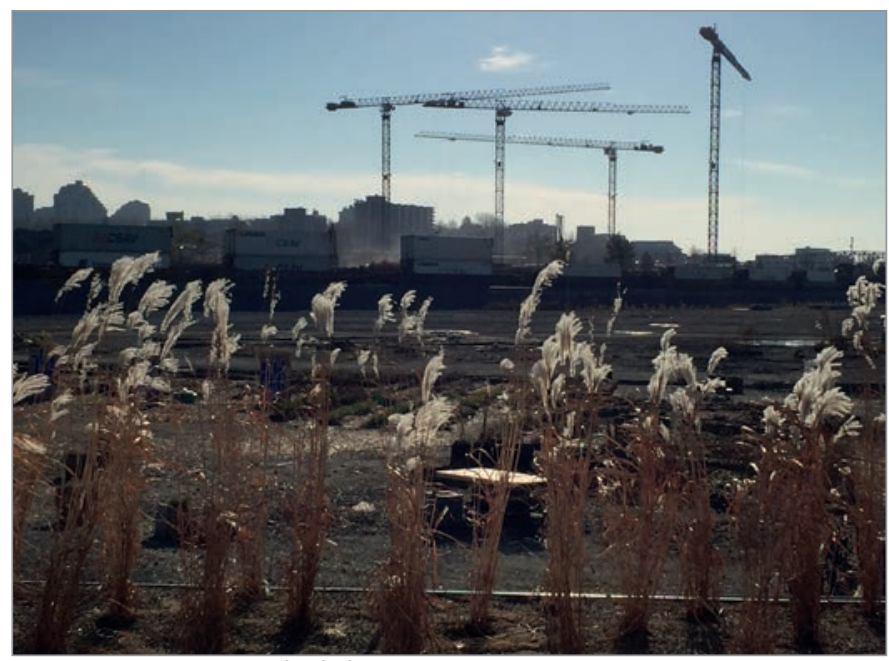

ILL. 3. LE SITE DES PROJETS ÉPHÉMĖRES, 2016. | MARYLÈNE PERRAS.

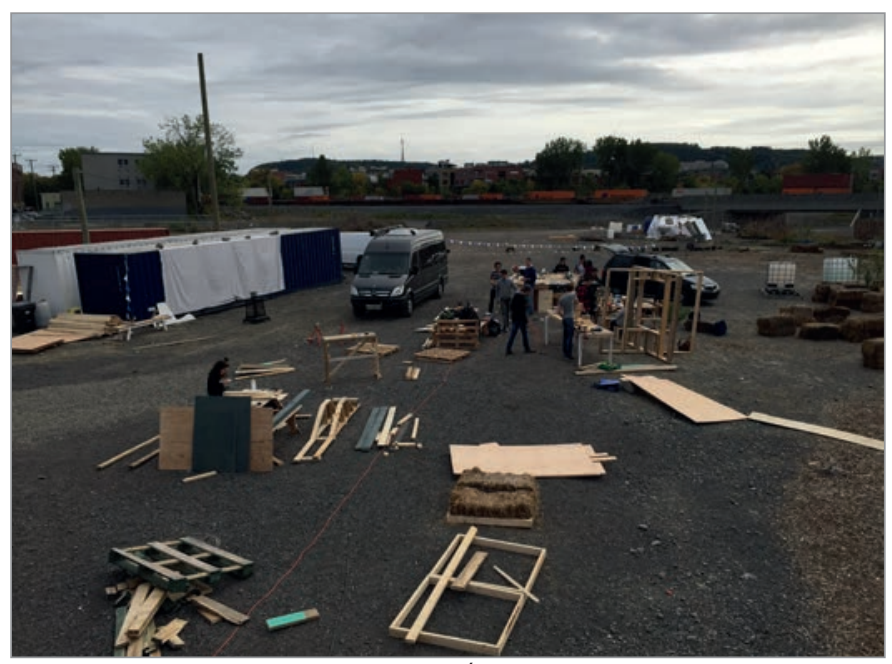

ILL. 4. ATELIER DE COCONSTRUCTION AVEC LES ÉTUDIANTS. | JONATHAN CHA. culturelles. II est situé à l'angle des avenues Atlantic et Durocher au cœur de l'île de Montréal, sur le site du futur campus MIL de l'Université de Montréal.

\section{L'INTERVENTION TRANSFORMATIVE DES ESPACES PUBLICS}

Cet article vise, à partir du prototype du Virage à Montréal, à poser les bases d'un nouveau type d'espace public répondant aux défis de la ville contemporaine. De nombreuses études, dont celle d'Aurélie de Smet en 2013'1, ont démontré que l'usage temporaire d'espaces urbains dont la fonction future n'est pas déterminée peut contribuer à l'élaboration d'un mode de développement de l'espace plus actuel, plus flexible et plus spontané. Partant de ce constat, ces espaces peuvent-ils se pérenniser sans se formaliser et instituer pour les instances municipales une nouvelle catégorie distincte de celles connues: parcs, places, promenades, squares, etc. ? Cet article offre des pistes de réflexion sur de nouvelles formes émergentes d'espaces publics plus libres et participatifs. Il explore le processus par lequel le prototype du Virage et des projets éphémères s'est approprié une parcelle d'une friche industrielle et a activé une réflexion sur notre manière actuelle de penser, construire et vivre les espaces 


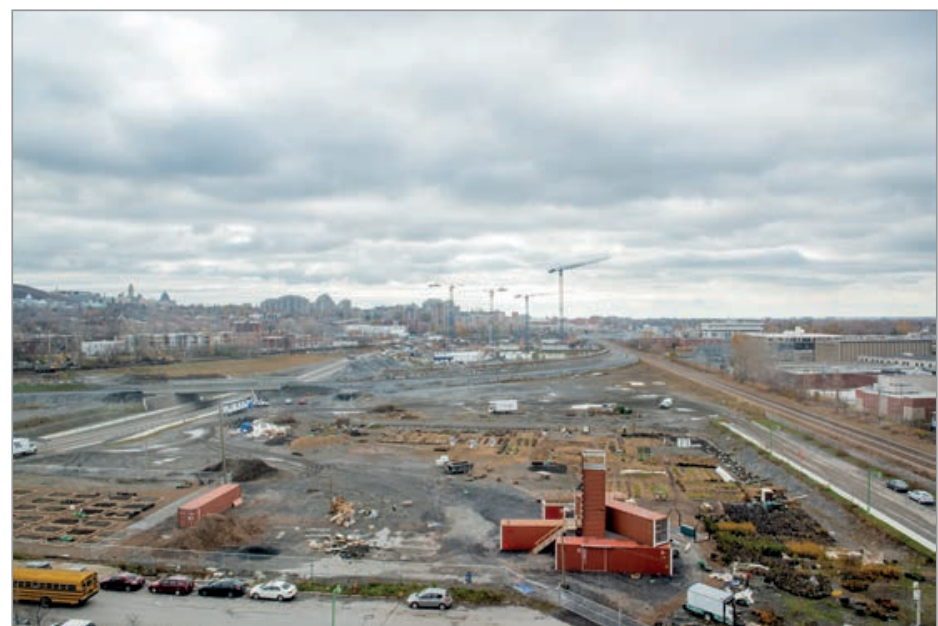

ILL. 5. LE SITE DES PROJETS ÉPHÉMÈRES, DU VIRAGE ET DU CAMPUS MIL. | CAMPUS MIL, UNIVERSITÉ DE MONTRÉAL.

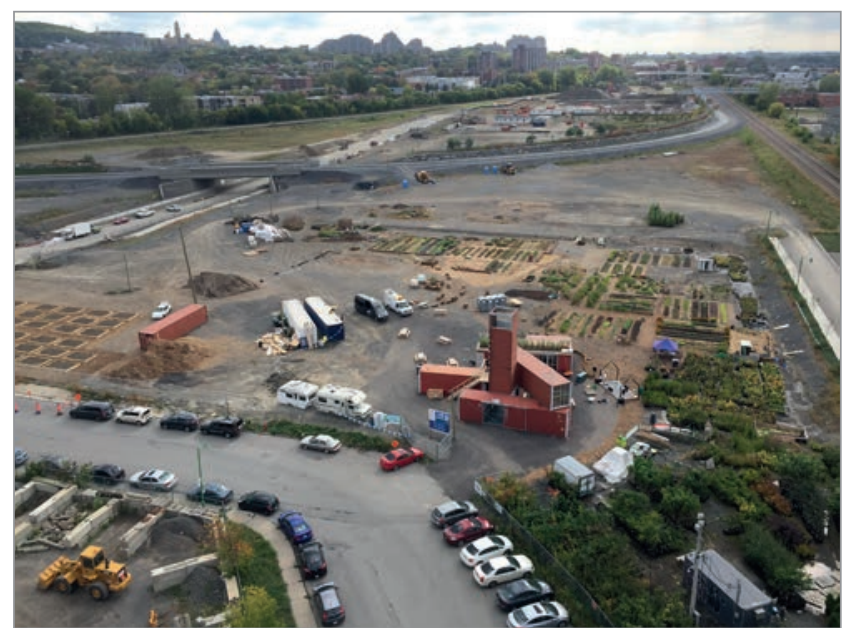

ILL. 6. LE SITE DES PROJETS ÉPHÉMÈRES, DU VIRAGE ET DU CAMPUS MIL. | JONATHAN CHA. publics². Des leçons pourront être tirées de cette expérimentation particulière qui n'a pas été sans défis et enjeux.

La réflexion sur les espaces publics porte de plus en plus vers les actions et les lieux temporaires et éphémères qui permettent une appropriation, une négociation et une construction collectives de sites postindustriels à l'avenir incertain. Ces "espaces inutiles ${ }^{3}$ ", abandonnés, sousutilisés ou en attente d'une fonction, portent en eux le pouvoir de transformer la manière de faire et de vivre les espaces publics, d'imaginer et de tester des formes d'occupations potentielles. C'est sous le vocable d'«urbanisme tactique» utilisé dès 2010 et popularisé par Mike Lydon et Anthony Garcia en 2015 dans leur ouvrage phare Tactical Urbanism4 ${ }^{4}$, que se regroupe une variété d'initiatives citadines spontanées de transformation des espaces publics urbains ${ }^{5}$. L'urbanisme tactique est ainsi défini comme des interventions temporaires et peu coûteuses en milieu urbain destinées à améliorer localement les milieux de vie des quartiers et à favoriser l'activation des lieux de rassemblement des villes (par des installations et une appropriation citoyenne). Il s'agit d'une approche délibérée et progressive pour inciter au changement, qui offre des solutions répondant à des défis de planification locale, qui propose un engagement à court terme, des attentes réalistes, qui a des risques faibles avec une récompense éventuellement élevée et qui permet un développement du capital social entre les citoyens et le renforcement des capacités organisationnelles entre les institutions publiques et privées, les organisations à but non lucratif et leurs partenaires. L'urbanisme tactique porte en lui un engagement politique qui le distingue de I'urbanisme temporaire orienté vers une temporalité et l'urbanisme participatif guidé par une concertation citoyenne tout au long du processus de projet.

Les nombreuses déclinaisons typologiques de I'urbanisme tactique, du Park(ing) Day initié par le collectif REBAR ${ }^{6}$ de San Francisco au Do-It-Yourself Urbanism, ont été explorées par Nicolas Douay et Maryvonne Prévot ainsi que Pascale Nédélec ${ }^{7}$ et permettent de constater et de comparer les nouvelles modalités de fabrique des espaces publics depuis un peu plus d'une décennie. La pluralité des appellations de ces formes novatrices d'un urbanisme dit alternatif ou itératif regroupe trois orientations communes: les temporalités, I'engagement politique et l'action citoyenne ${ }^{8}$. Cet urbanisme propose de nouvelles façons de concevoir et de planifier l'espace ${ }^{9}$ et de rendre compte de son pouvoir transformateur sur le lieu, le quartier et la vie urbaine ${ }^{10}$. Les expérimentations qui en découlent s'inscrivent dans le processus évolutif des villes, sous le régime d'austérité budgétaire et de sous-investissements dans les espaces publics et plus largement dans un contexte de crise - écologique, sanitaire, sociale et politique - de l'urbanisme planétaire ${ }^{11}$.

Si l'origine de l'urbanisme tactique peut en être une de la revendication ou de la négociation collective, d'autres I'approchent comme un "urbanisme d'entente, un terrain d'entente ${ }^{12} »$. "L'intervention tactique ne se substitue pas aux espaces traditionnels, mais en devient un complément, elle doit être comprise comme une manière engagée de comprendre, révéler et de faire - autrement - l'espace public contemporain $^{13}$. " Ces pratiques de gestion transitoire d'espaces urbains en friche qui 


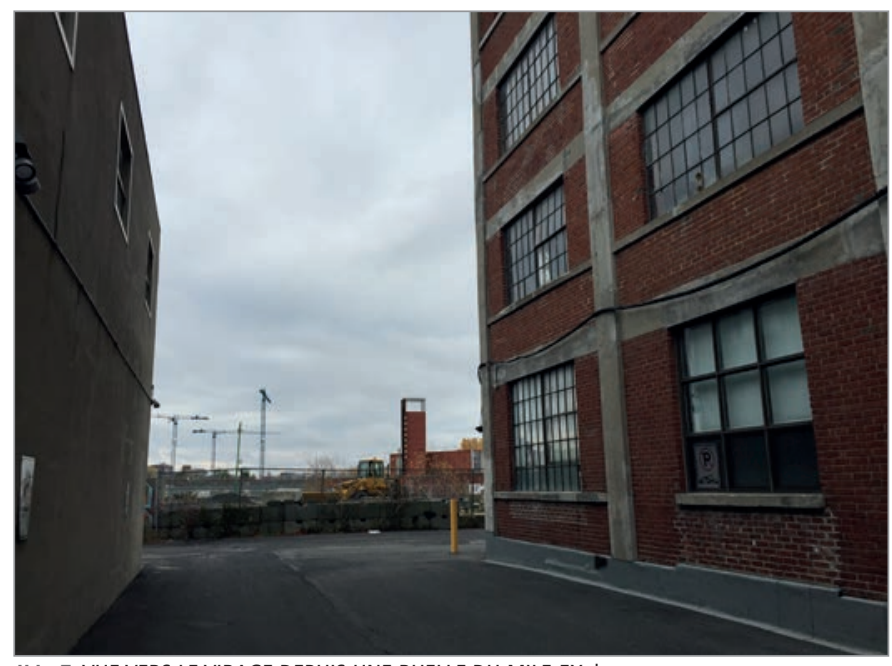

ILL. 7. VUE VERS LE VIRAGE DEPUIS UNE RUELLE DU MILE-EX. | JONATHAN CHA.

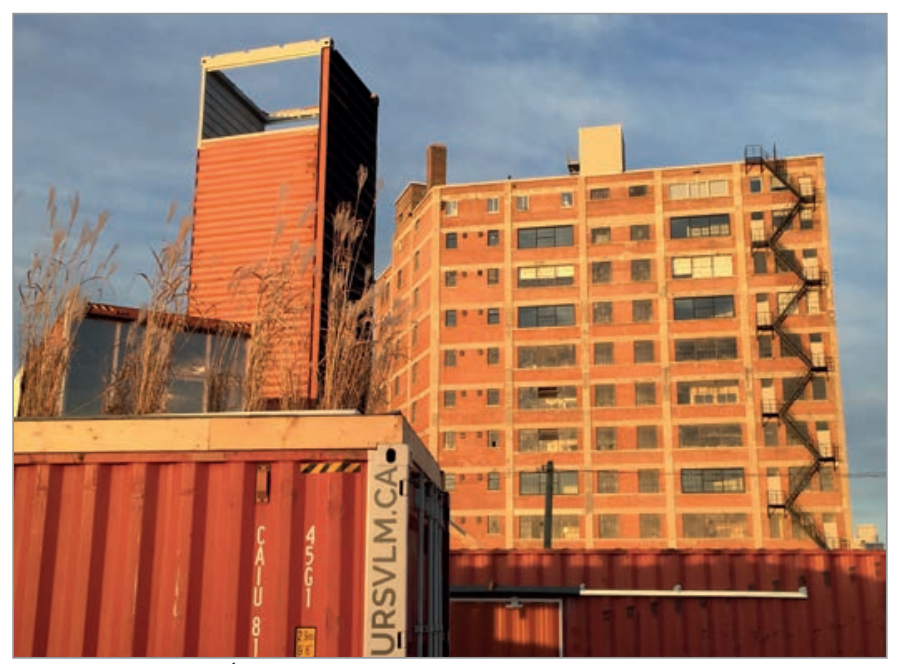

ILL. 8. LE VIRAGE ET L'ÉDIFICE DU 400, AVENUE ATLANTIC. | JONATHAN CHA. apparaissent dans la ville néo-libérale créative et participative ${ }^{14}$ sont des indicateurs de nouvelles formes de citoyenneté ${ }^{15}$ et illustrent l'attention portée aux espaces publics et à ses appropriations citoyennes en temps de crise. Les projets temporaires remplissent ainsi le vide entre une économie urbaine changeante et un paysage urbain changeant ${ }^{16}$. Selon Florian Haydn et Robert Temel, "les usages temporaires sont ceux qui cherchent à tirer des qualités uniques de I'idée de temporalité ${ }^{17}$ ». Ils deviennent ainsi, pour Geneviève Vachon, Érick Rivard et Alexandre Boulianne, des indicateurs d'une nouvelle manière d'occuper et de construire l'espace public et des "révélateurs d'enjeux urbains, de lieux sous-utilisés et d'actions transformatives concrètes $^{18}$ ". Le prototype du Virage $s^{\prime}$ inscrit dans cette dynamique, celle qui admet la crise de l'espace public et qui accepte que "le projet est l'affaire d'un nombre croissant de personnes, intervenant à tout moment d'un processus qui n'est plus linéaire ${ }^{19}$ ». Pour Denis Delbaere, cet objet auto-institué compromet l'espace public comme forme. La forme n'est plus ce qui doit advenir, mais est déjà là, l'espace public est à penser comme déjà là, comme donné d'emblée. Ces interventions transformatives comme celle du Virage participent dès lors à l'émergence de nouvelles définitions et formes d'espaces publics.

\section{UN ENTRE-LIEU TRANSITOIRE}

Ces terrains libres que I'on qualifierait de vagues ${ }^{20}$ ou de paysages en pause ${ }^{21}$ sont d'«immenses réservoirs d'espaces encore inexploités par l'imagination ", pour reprendre l'expression de William $\mathrm{H}$. Whyte ${ }^{22}$. Leur condition incertaine offre la possibilité de prototyper un nouveau genre d'espace ne répondant pas aux critères traditionnels de formes, d'équipements et d'usages : "l'indétermination laisse de la place pour une appropriation créative et des moyens alternatifs d'expérimenter la ville ${ }^{23}$ ». L'activation ${ }^{24}$ de ces espaces temporaires proposant des expériences communautaires, culturelles, éducatives ou festives en marge change la perception et accélère la réflexion sur le rôle, la forme et la gouvernance des espaces publics urbains. Ces interventions créent des réseaux d'espaces publics alternatifs en parallèle des trajectoires connues dans le contexte de transformation des villes «intermittentes ${ }^{25}$ ». Un aménagement temporaire consiste à intégrer temporairement des pratiques et des usages quotidiens dans un temps défini sans agir sur la suite du projet. Une occupation transitoire se définit comme une action visant à tester, à questionner et à expérimenter des pratiques sur un site pour préfigurer son aménagement futur ${ }^{26}$. L'éphémère ou le transitoire est explicite, intentionnel et d'une temporalité limitée. Il porte en lui la volonté d'ouvrir les horizons des possibles. Le Virage se positionne comme un «entre-lieu transitoire ${ }^{27}$ ", un espace interstitiel où des processus actifs «de dépassement et de régénération ${ }^{28}$ " participent au devenir du lieu ${ }^{29}$. Le concept d'entre-lieu tire son origine dans l'étude du lieu (non-lieu, hyper-lieu, tiers paysage, etc.). Par ses actions créatives, le Virage génère un état "entre " qui révèle peu à peu le potentiel du lieu, qui transforme sa perception de non-lieu à lieu d'espoir et d'attente. L'espace transitoire du Virage, qui habite l'espace en transformation, corollaire de la notion d' « intermittence" de Claudia Faraone et Andrea Sarti, s'inscrit dans cette réalité de dissolution formelle de l'espace public ${ }^{30}$. 


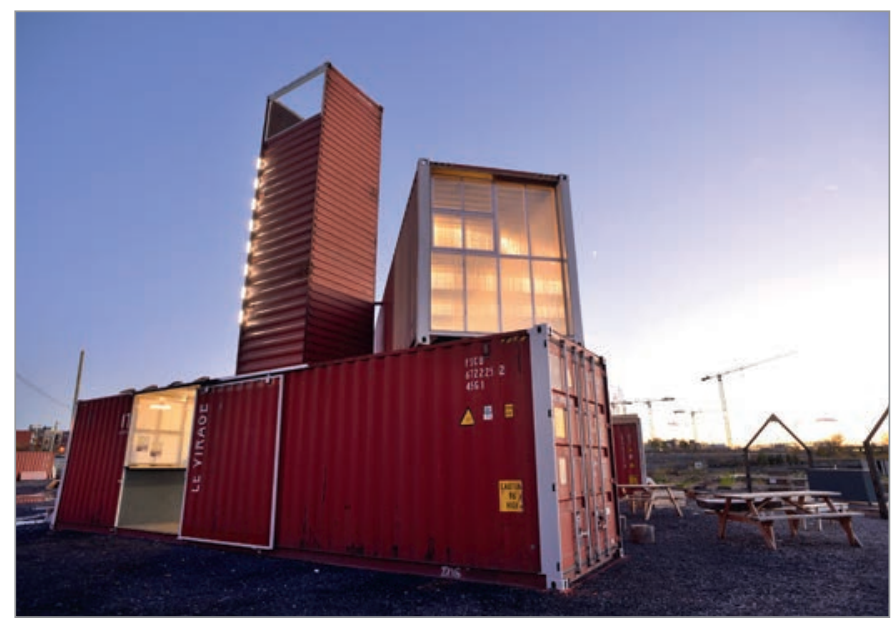

ILL. 9. LE VIRAGE ET L'ARCHITECTURE LUMINEUSE DES CONTENEURS COMME «SIGNAL » URBAIN. | MARYLÈNE PERRAS.

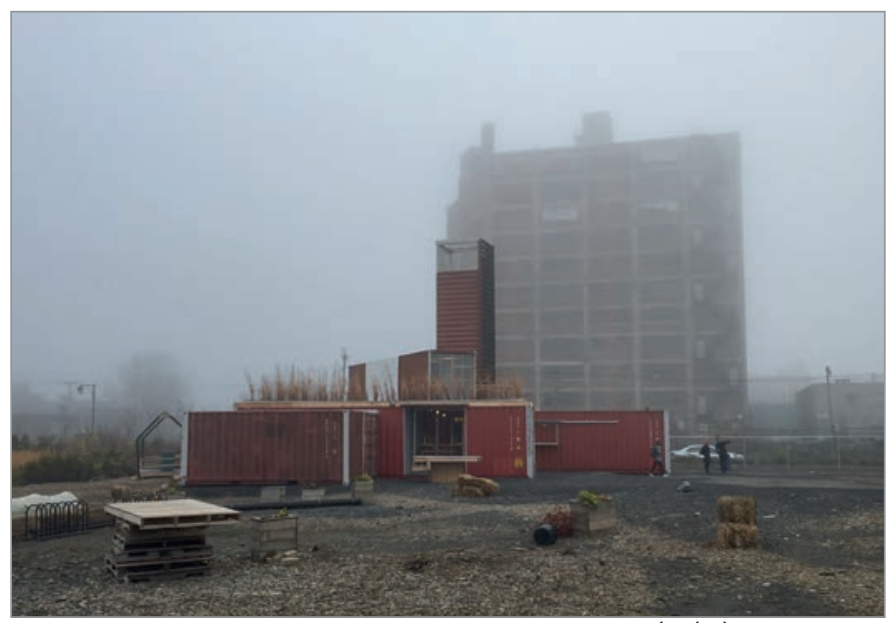

ILL. 10. L'AMBIANCE DE PAYSAGE INDUSTRIEL DU SITE DES PROJETS ÉPHÉMÈRES, DU VIRAGE ET DE L'ÉDIFICE DU 400, AVENUE ATLANTIC. | MARYLÈNE PERRAS. de Smet définit trois groupes d'acteurs et d'objectifs menant à la réalisation d'espaces temporaires: I'opportunisme (qui profite d'une situation spécifique), I'activisme (qui milite pour une cause en adoptant une attitude critique) et I'auto-organisation (qui porte l'idéologie de produire une ville meilleure par une approche bottom up). Le Virage ${ }^{31}$ répond particulièrement aux premier et troisième groupes et pourrait être «un exemple d'urbanisme temporaire qualifié d'assemblages précaires d'expressions matérielles et identitaires ${ }^{32}$ ". Le Virage n'est ni militant ni revendicateur, mais s'inscrit néanmoins dans la remise en cause de l'urbanisme néolibéral ${ }^{33}$. Approuvé d'entrée de jeu par des institutions publiques, il est né de l'opportunité d'investir une parcelle de terrain appartenant à l'Université de Montréal sur son futur campus MIL et d'un financement obtenu de la Ville de Montréal en vertu du Plan de développement urbain, économique et social (PDUES) des secteurs Marconi-Alexandra, Atlantic, Beaumont et De Castelnau. C'est ainsi qu'un terrain expérimental contrôlé et un financement ont été obtenus ${ }^{34}$. La démarche a été initiée et réalisée conjointement entre
I'organisme Montréal ville en mouvement $(\mathrm{MVM})^{35}$ et le Laboratoire des récits du soi mobile rattaché au Département de littérature de la Faculté des arts et sciences de I'Université de Montréal. La multidisciplinarité s'est imposée dès le départ avec des collaborateurs universitaires et professionnels des milieux de l'aménagement, du cinéma et des lettres. Les médias ont même présenté le Virage comme «une place publique pour rapprocher le public du savoir scientifique ${ }^{36} »$. MVM est un organisme sans but lucratif (OBNL) qui s'intéresse à la participation active des citoyens dans la réflexion sur le devenir de la ville et des espaces publics. Il vise à certains égards «l'empowerment citoyen par l'appropriation et la prise en charge de l'espace public ${ }^{37}$ '». Inauguré en 2016, le Virage rejoint sur le même site les projets éphémères amorcés en 2015, composés de parcelles de terrains offertes à des organismes locaux qui y pratiquent l'agriculture urbaine. Le Virage, que j'ai cocréé et où j'ai agi simultanément comme acteur de changement et chercheur, est un lieu d'expérimentation démocratique tant dans les contenus offerts sur l'environnement urbain que dans les interactions, les cohabitations et les pratiques de l'espace.
J'ai ainsi contribué à construire, gérer, opérer et programmer le Virage tout en profitant de cette expérience pour penser la contribution de nos décisions, observer les occupations et évaluer sa portée sur la réflexion des espaces publics. Cet article est le fruit de ce travail de terrain et intellectuel.

\section{LE PROTOTYPAGE URBAIN}

La notion berlinoise de catalyseur urbain, antérieure à celle d'urbanisme tactique (théorisée notamment par Misselwitz, Oswalt et Overmeyer ${ }^{38}$ ), a inspiré la démarche de prototypage. Pour le collectif allemand Urban Catalyst Studio, le temporaire doit intégrer le discours sur le design urbain, l'usage temporaire servant de catalyseur urbain pour une transformation urbaine. Le catalyseur urbain se définit ainsi comme des éléments ou des actes d'une durée limitée qui initient des processus qui sont appelés à se transformer ou à disparaître. Cet outil questionne les manières de faire, interroge l'identité et l'intégrité du lieu. Les utilisateurs deviennent alors des défricheurs, des pionniers. Selon I'Urban Prototyping Research Lab fondé il y a une décennie à 


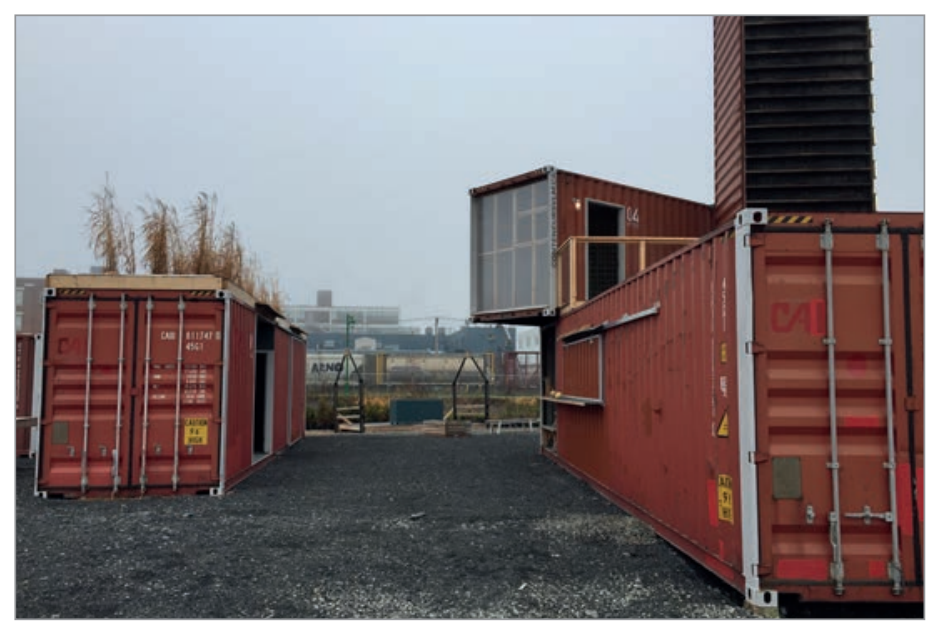

ILL. 11. LE CEEUR DE L'ASSEMBLAGE D'ESPACES ET DE VOLUMES DU VIRAGE ET LES TRAINS DE MARCHANDISES EN MOUVEMENT. | MARYLĖNE PERRAS.
ILL. 12. LE PHARE DU VIRAGE, ÉLÉMENT D'ARCHITECTURE VERTICALE QUI DIALOGUE AVEC LA TOUR DE L'UNIVERSITÉ DE MONTRÉAL. | MARYLÈNE PERRAS.

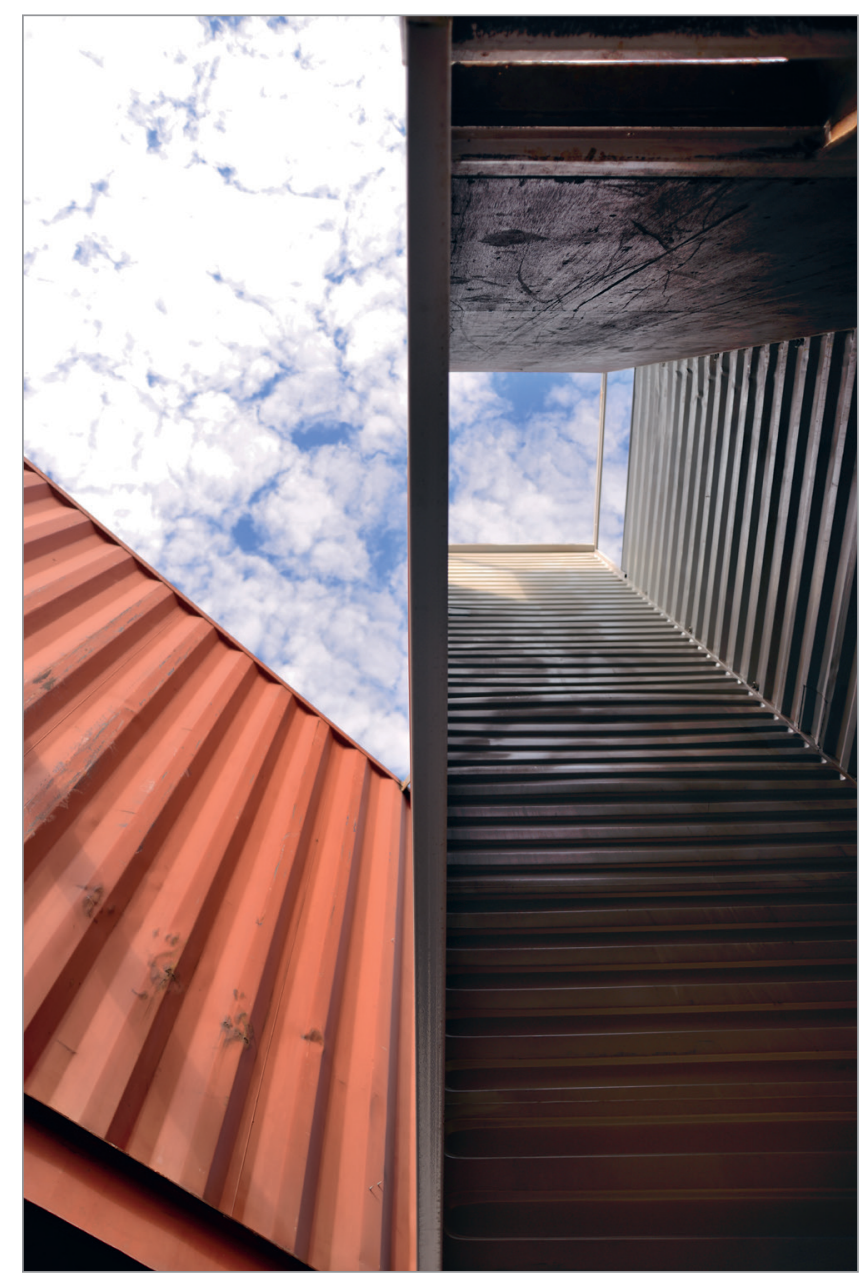

San Francisco, le prototypage urbain est un mouvement mondial qui explore comment le design participatif peut améliorer les villes ${ }^{39}$. Selon la firme Gehl Architects,

We have to develop new models for conceiving, testing, financing and implementing projects that respond to this reality. Rapid urban prototyping, pilot projects, and the tactical urbanism approach are the first steps at addressing the speed in which the way we move, meet, and spend time in cities is changing 40

Le prototypage du Virage est une forme expérimentale d'urbanisme au service des «espaces-enjeux ${ }^{41}$ ». Le Virage et les projets éphémères s'inscrivent dans un processus de prototypage, une tendance s'observant à l'échelle occidentale qui privilégie une action rapide et participative dans la ville ${ }^{42}$. Le prototypage n'est pas un concept nouveau et est emprunté à une variété de sphères (urbanisme, design d'objets, construction, informatique, gouvernance, etc.). Le prototype est le modèle premier, le modèle original. Sa racine grecque renvoie à une forme primitive, à une première impression. Ce premier exemplaire est par définition incomplet et non définitif, "a working model, albeit crude and incomplete, speedily constructed ${ }^{43}$ ». Il est une matérialisation d'une idée pour tester un concept ou un processus. Dans le cas présent, il est l'étape initiale, la phase bêta d'un cycle de réflexion et de production qui ne mène pas à une volonté de reproduction. Le but visé par le Virage - et les projets éphémères - n'est pas tant de le répliquer tel quel que d'apprendre de celui-ci, de le faire évoluer, comme le démontrent ses phases successives d'évolution. Nous rejoignons ainsi I'auteure Christiane Floyd qui définissait le prototypage comme "ongoing, suggesting an erosion of the boundary between prototype and final object $^{44}$ ». Le prototype doit être compris 


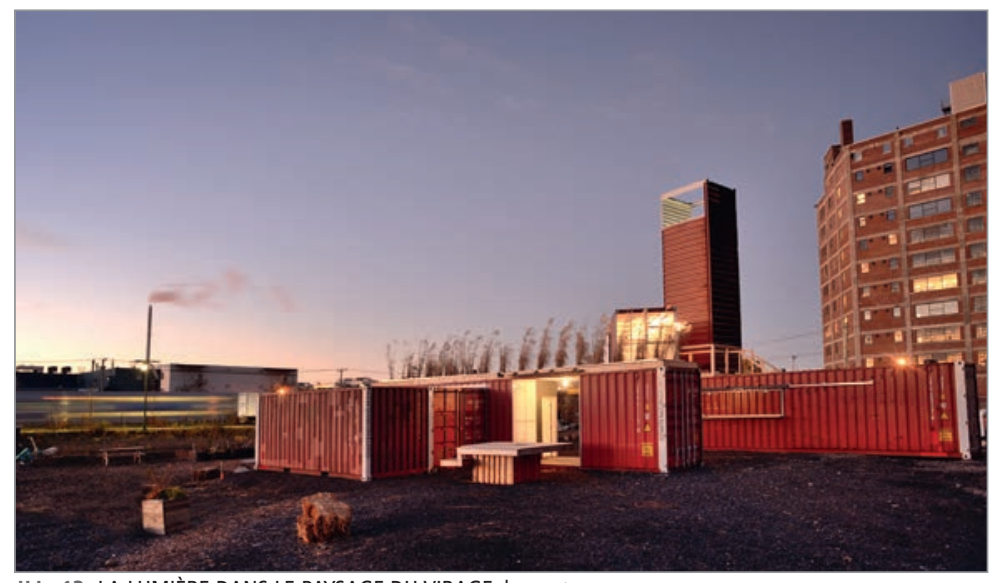

ILL. 13. LA LUMIĖRE DANS LE PAYSAGE DU VIRAGE. | MARYLĖNE PERRAS.

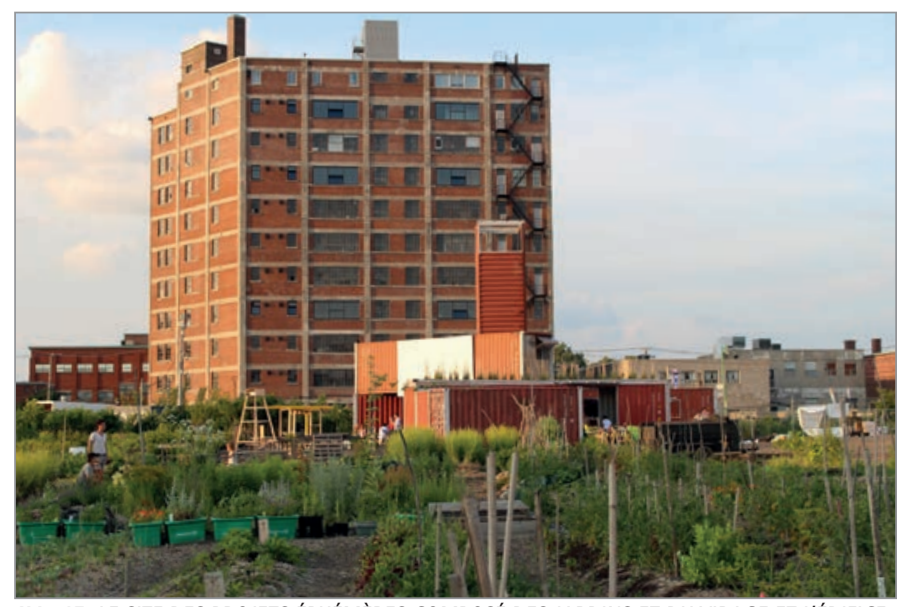

ILL. 15. LE SITE DES PROJETS ÉPHÉMĖRES COMPOSÉ DES JARDINS ET DU VIRAGE ET L'ÉDIFICE DU 400, AVENUE ATLANTIC. | CAMPUS MIL, UNIVERSITÉ DE MONTRÉAL

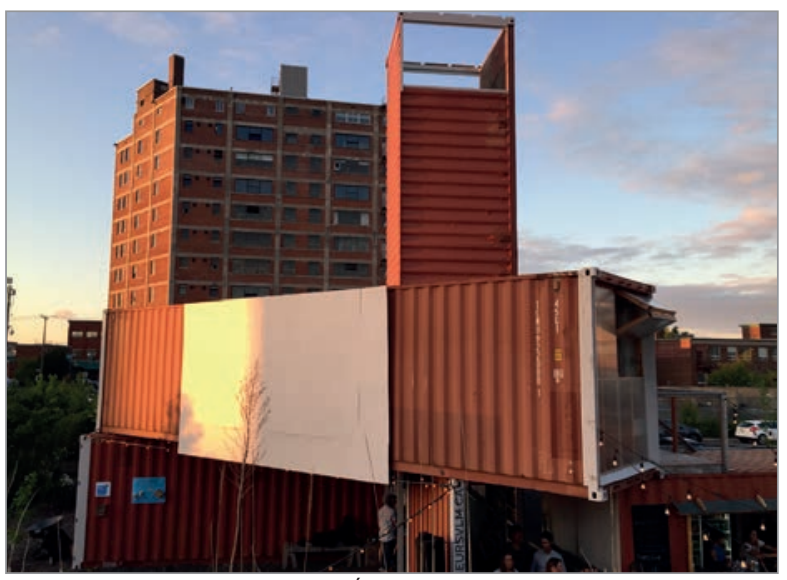

ILL. 14. LE VIRAGE ET LA CONTINUITÉ DES ARCHITECTURES. | JONATHAN CHA.

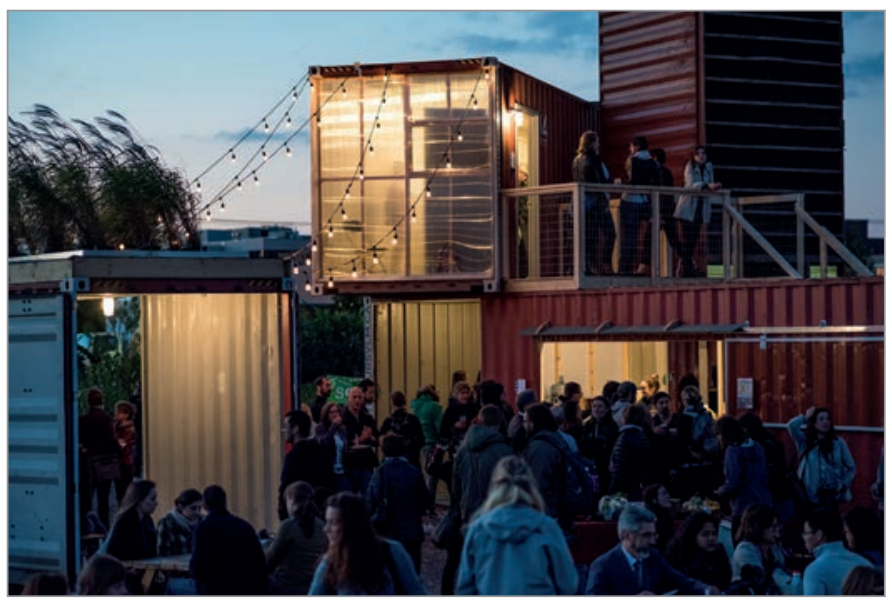

ILL. 16. LE VIRAGE. | MONTRÉAL VILLE EN MOUVEMENT, MATHIEU DESHAYES. dans cette étude de cas comme "things we make to find out things ${ }^{45}$ ". Dans I'approche associée à la fabrique de l'espace public, le prototypage présente des défis intéressants à mettre en œuvre par les praticiens et à étudier par les chercheurs. Le prototypage du Virage peut être compris comme « un artefact précurseur holistique du produit final ${ }^{46}$ " et encore plus comme «la permission d'expérimenter ${ }^{47}$ ", puisqu'il est implanté sur la propriété de I'Université de Montréal qui a conclu des ententes pour permettre à I'OBNL MVM d'utiliser ses terrains. Le prototype du Virage cherche à repenser notre manière de concevoir et de pratiquer l'espace public, d'activer et donner sens à un lieu et d'étudier les attitudes sociales face à l'espace et les influences du temporaire et de l'informel sur la création de lieux collectifs.

La temporalité du prototype est importante puisque l'entente d'occupation convenait d'un terme de cinq ans (20162020) pour le Virage et d'un terme de un an renouvelable chaque année pour les projets éphémères. Le prototypage peut donc évoluer et être considéré comme "lent", ce qui offre davantage de marge de manœuvre pour l'exploration. Les auteurs Lucy Kimbell et Jocelyn
Bailey affirment que «le prototypage lent permet d'explorer l'adaptabilité et l'adaptation d'une idée à un lieu et à un groupe de personnes donnés, tout en laissant le temps d'établir des infrastructures sociales et d'explorer les problèmes de manière collective ${ }^{48}$. " Jan Gehl aborde la notion de "permanence du temporaire" et demande : comment réinventer les notions de temporaire et de permanent pour faire en sorte que les environnements urbains évoluent en phase avec l'évolution rapide des cultures urbaines? L'aménagement urbain qui se réalise rapidement, qui évolue au gré des besoins quotidiens et en temps réel, est une 


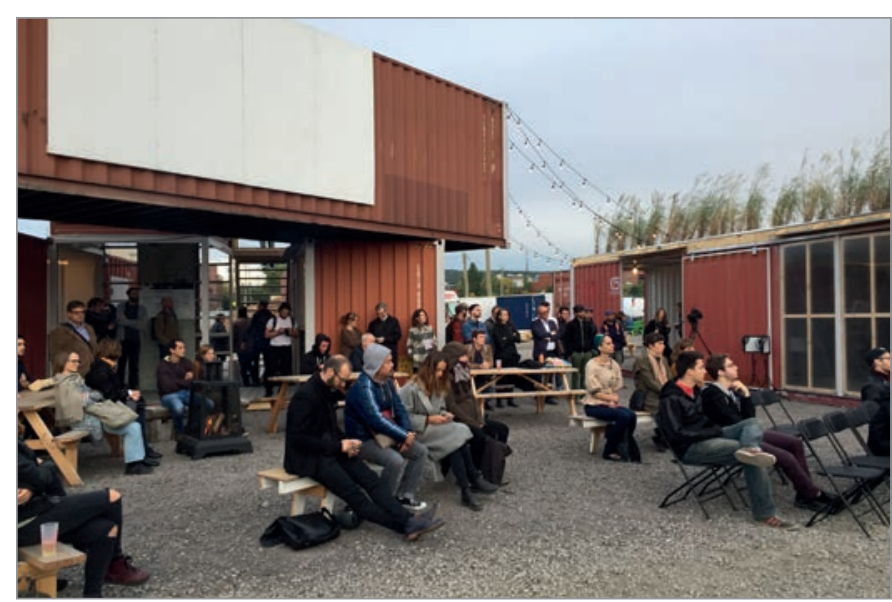

ILL. 17. UNE CONFÉRENCE EXTÉRIEURE DANS L'AGORA DU VIRAGE. | JONATHAN CHA.

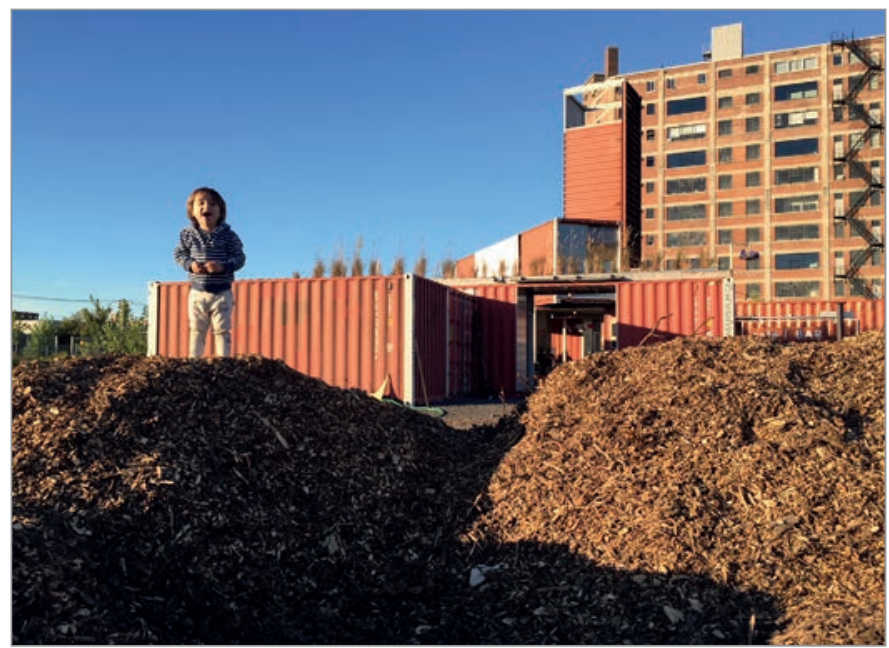

ILL. 19. AIRE DE JEU INFORMELLE AU VIRAGE POUR LE PLAISIR DES TOUT PETITS. | JONATHAN CHA.

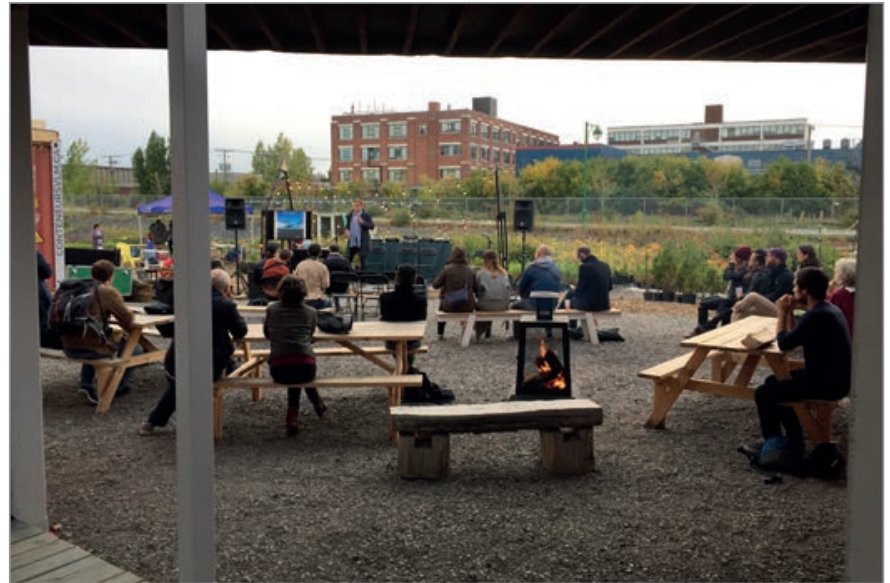

ILL. 18. UNE CONFÉRENCE EXTÉRIEURE DANS L'AGORA AU VIRAGE VUE DEPUIS LE PASSAGE COUVERT. | JONATHAN CHA.

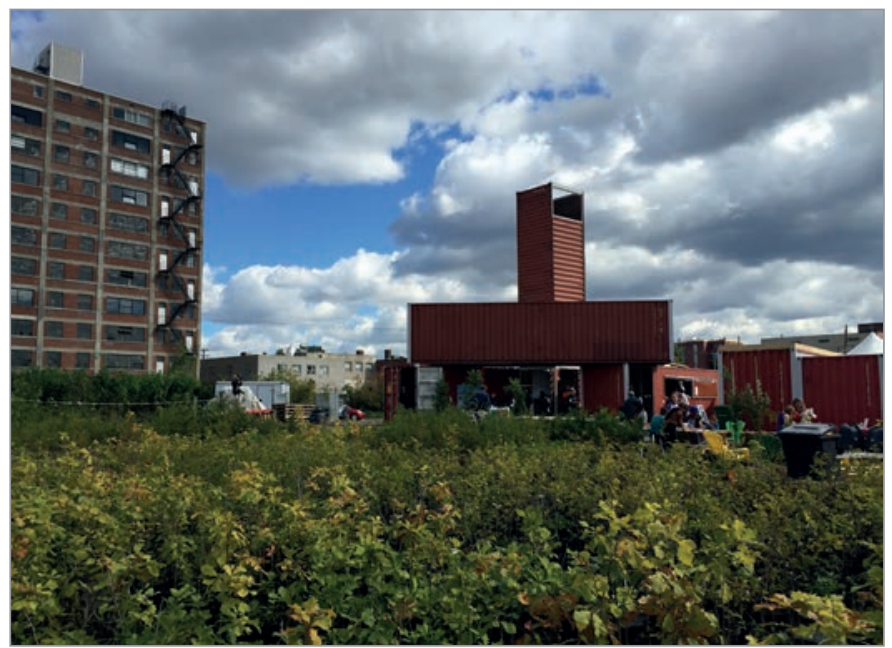

ILL. 20. LES JARDINS ÉPHÉMÈRES ENTOURANT LE VIRAGE. | JONATHAN CHA. réalité et une attente grandissantes pour la population. Le prototypage du Virage et des projets éphémères dépasse les limites de la pratique urbanistique habituelle. Il offre à l'Université de Montréal et à la Ville de Montréal la possibilité « d'intervenir entre les réalités et les potentialités », la flexibilité dans la planification et l'élaboration des politiques, tout en gardant les choses ouvertes, tandis que des solutions provisoires sont anticipées, développées ou rejetées ${ }^{49}$.

Le prototypage est, par nature, une situation nouvelle comportant un haut niveau de plaisir et de risque. Le prototype, c'est alimenter la réflexion sans la peur de l'échec; en effet, le Virage a testé plusieurs types d'activités et sources de financement sans avoir réussi à assurer une rentabilité. Le prototype n'est ni une forme ni un lieu, mais un concept, un modèle d'expression qui subit des mutations constantes. "Le projet en soi est un prototype qui teste l'aménagement ${ }^{50}$." Le Virage s'est enrichi chaque année de nouvelles structures pour répondre à l'évolution de sa programmation et de sa réflexion sur l'espace (notamment l'ajout d'un hall, d'espaces d'exposition, d'un mur-écran de projection, d'une terrasse couverte, d'espaces plantés, de mobilier).
Comme énoncé plus haut, le prototypage du Virage et des projets éphémères s'inscrit dans la démarche de l'urbanisme tactique qui mise sur des interventions à petite échelle et temporaires dans l'espace public pour améliorer une condition urbaine et initier des changements à long terme dans l'aménagement de la ville. En même temps, il la dépasse par ses intentions de réflexion sur la création, l'occupation et la gestion des espaces à caractère public et sur la recherche d'une nouvelle forme d'espace public à inventer. L'exemple barcelonais est une source d'inspiration intéressante à cet égard. La mairie de Barcelone 


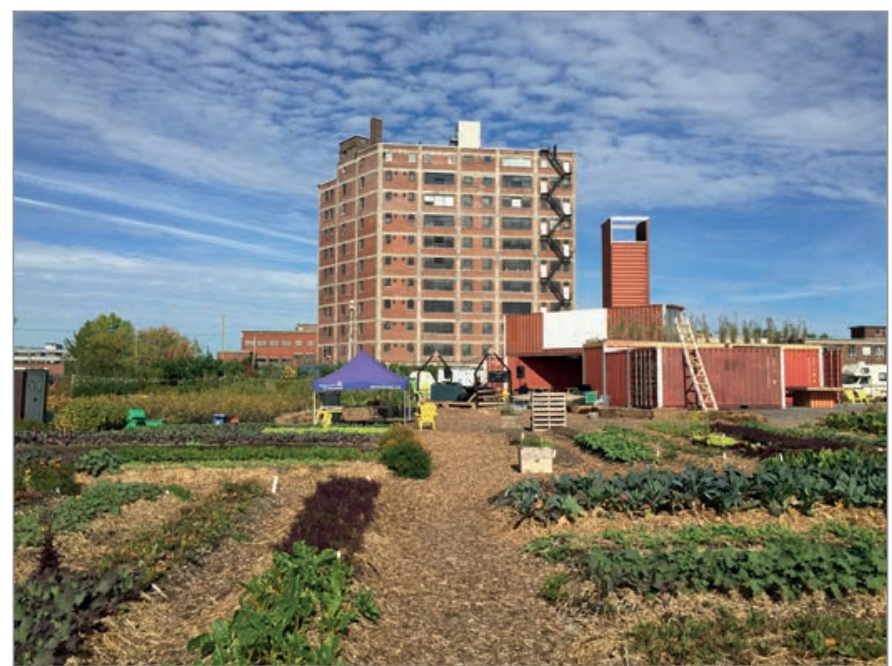

ILL. 21. LES JARDINS ÉPHÉMĖRES ENTOURANT LE VIRAGE. | JONATHAN CHA.

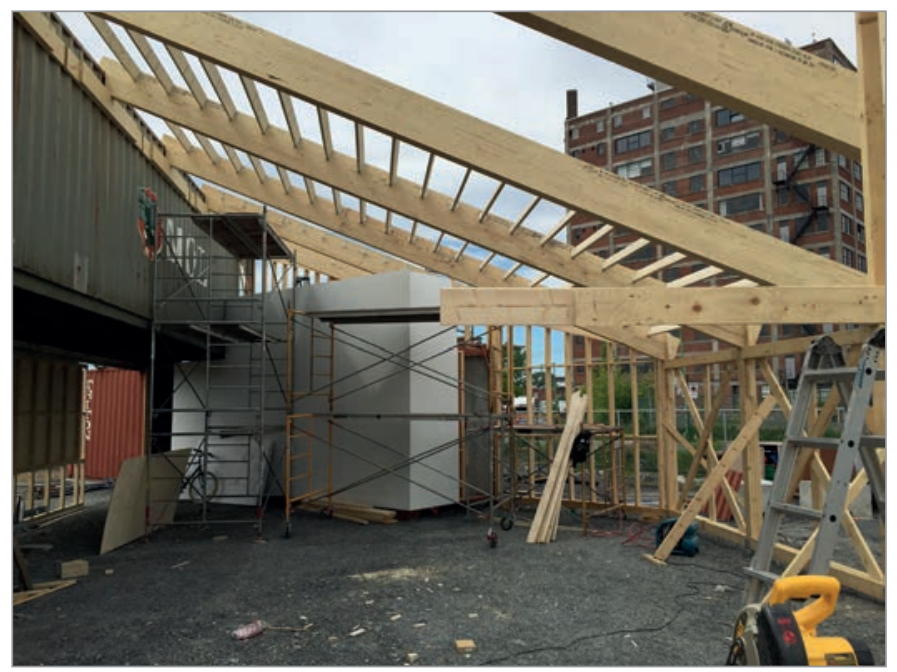

ILL. 22. LE GRAND HALL DU VIRAGE EN CONSTRUCTION. | JONATHAN CHA. cède gratuitement [d]es espaces à des collectifs et à des associations, recensés dans le fichier municipal des entités citoyennes sans but lucratif (Fitxer General d'Entitats Ciutadanes], pour qu'elles les aménagent en y implantant des activités d'intérêt public ou d'utilité sociale. Les activités proposées doivent être temporaires, se développer dans des structures provisoires et présenter un caractère éducatif, sportif, ludique, culturel ou artistique ${ }^{51}$.

La stratégie du Virage vise à prototyper, à valoriser et à communiquer un lieu jusqu'alors inconnu du public et à sensibiliser les citoyens au potentiel d'appropriation et d'occupation d'un espace. Le Virage est pensé " avant tout comme une machine dont la simplicité et l'aspect modulable permettent de nourrir une réflexion sur la ville, et de le faire hors des murs de l'université52 ". Le Virage et les projets éphémères donnent aussi sens à un lieu par une approche singulière et contextualisée liée à l'identité du site. Plusieurs auteurs évoquent d'ailleurs l'importance du génie du lieu et la possibilité de révéler un lieu par des actions ${ }^{53}$.

\section{LE PROCESSUS EXPLORATOIRE DU VIRAGE ET DES PROJETS ÉPHÉMĖRES}

Le prototype du Virage et des projets éphémères a recouru à une démarche originale qui a façonné ses formes et ses usages. Voici les principes par lesquels il est né et a évolué et comment il peut influencer les formes émergentes d'espaces publics. Rappelons que la stratégie visait à prototyper, valoriser et communiquer un lieu jusqu'alors inconnu du public et sensibiliser les citoyens au potentiel d'appropriation et de réclamation d'un espace. Le processus exploratoire a recouru à 1) la coconception et la coconstruction étudiantes, 2) la création d'un écosystème humain et écologique, 3) l'expérience citoyenne par la programmation culturelle, I'éducation et la formation, 4) I'adaptation, l'agilité et la flexibilité et 5) la liberté d'action citoyenne. Cet espace hérité à contrecourant des pratiques urbanistiques a accueilli des pionniers urbains engagés à créer des espaces démocratiques au bénéfice de tous et contribué à développer une nouvelle manière plus inclusive de construire et d'habiter l'espace public en devenir. Cette expérimentation riche en enseignements a rencontré plusieurs embûches qu'il convient de présenter, puisque parties inhérentes de ce processus particulier.

\section{1) La coconception \\ et la coconstruction}

Les jardins des projets éphémères sont réalisés par des organismes et des coopératives locaux engagés dans leur communauté. Ils s'inscrivent dans leur expertise et leur modèle d'affaires, bien que I'initiative de leur regroupement sur un terrain commun soit originale. Le Virage était porté à la base par une volonté de formation mettant à profit les compétences des disciplines de l'aménagement. Le processus créatif du Virage a valorisé les étudiants comme acteurs clés et mis de l'avant le concours et le workshop, l'accompagnement professionnel, la construction et l'exploration in situ, ainsi que la collaboration avec des partenaires de milieux variés. Toute la démarche de coconception s'est faite par un processus de design rigoureux impliquant une cinquantaine d'étudiants de toutes les écoles 


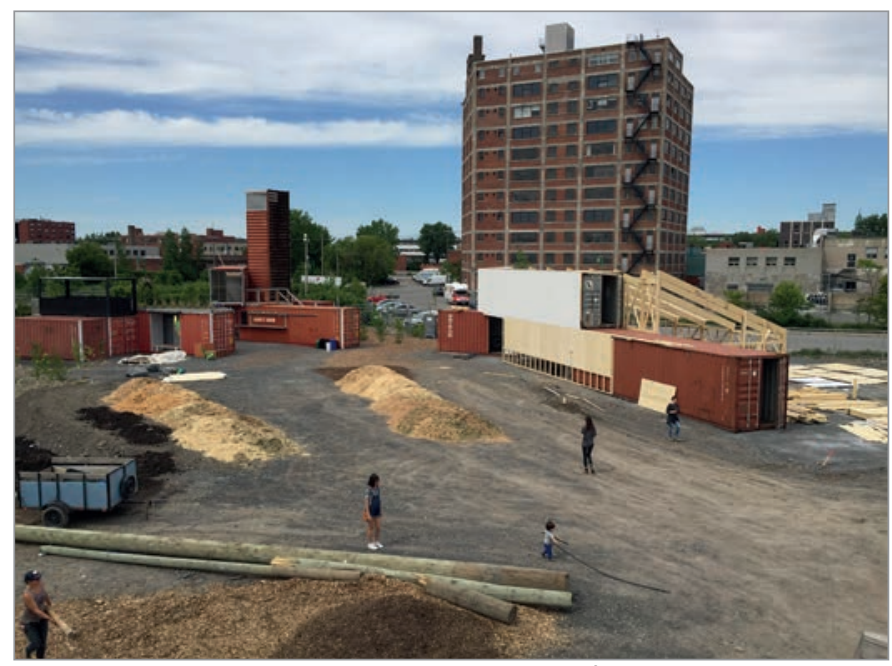

ILL. 23. LE GRAND HALL DU VIRAGE EN CONSTRUCTION ET L'ÉVOLUTION DES SURFACES AU SOL. | JONATHAN CHA.

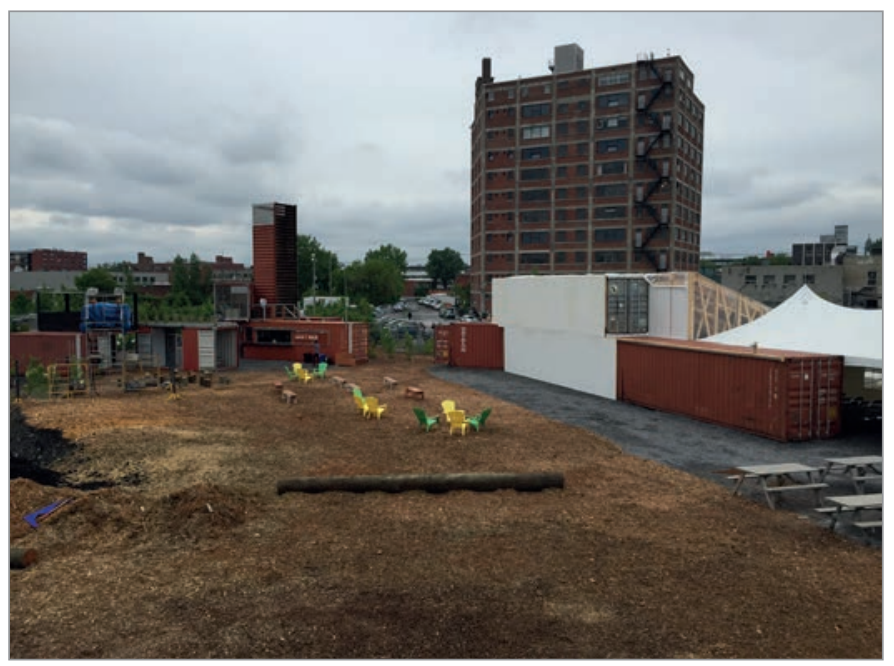

ILL. 24. L'ESPLANADE ET LE GRAND HALL DU VIRAGE. | JONATHAN CHA. de la Faculté de l'aménagement de l'Université de Montréal (architecture, architecture de paysage, design d'intérieur, design industriel, urbanisme). Dans des concours prenant la forme de workshops, des professeurs, des chargés de cours et des professionnels ont supervisé, jugé et sélectionné les propositions les plus originales et réalistes. Les étudiants ont par la suite été encadrés (sous forme de stages) par MVM et des bureaux professionnels et ont participé au processus de coconstruction du lieu en usine et in situ, en collaboration avec de jeunes diplômés et des entrepreneurs ${ }^{54}$. Ce type d'approche très structurée misant sur la multidisciplinarité et la formation n'est pas sans heurts (difficulté à trouver des étudiants motivés et disponibles, beaucoup de temps d'encadrement et de logistique, manque d'expérience des intervenants, difficulté d'avoir des professionnels expérimentés en raison des faibles ressources financières, collaboration nécessaire entre les acteurs, temps limité), mais a l'intérêt de passer rapidement de l'idée à la réalisation, de favoriser l'expérimentation et de forcer « les designers à vivre et à observer intensément le site du projet ${ }^{55} »$.
Le processus exploratoire d'édification de I'espace mise sur une expérimentation à l'échelle 1:1 et sur une mise en contact avec les réalités d'un projet (techniques, financières, d'échéancier). Le nom et l'implantation du Virage, nom du concept lauréat, s'inspirent de l'ancienne rotonde et de la cour de triage du Canadien Pacifique de même que de la courbe que forment les avenues Durocher et Atlantic. Il était important de faire sens et de faire écho au territoire ${ }^{56}$. II adopte une approche contextualiste, recourt à des matériaux réutilisés (les conteneurs) et à une esthétique brute et industrielle qui correspond au génie du lieu de l'ancienne emprise ferroviaire et de ses abords. Les conteneurs rougeâtres dialoguent directement avec l'édifice emblématique voisin et les trains transportant des conteneurs qui traversent de biais le site. Par sa matérialité, ses courbes et ses cadrages, le Virage dialogue autant avec la forme urbaine, les dynamiques de mouvements des trains de marchandises et des voies ferrées, l'édifice industriel du 400, avenue Atlantic, qu'avec les vues dirigées et ouvertes vers le mont Royal et le campus de la montagne de I'Université de Montréal. MVM a opté pour cette méthode, de la première phase de construction du Virage en 2016 à la dernière phase d'agrandissement en 2018. La coconception et la coconstruction comme approches de transformation de la ville sont une manière originale de poser un diagnostic, de révéler des potentiels, et de faire de l'espace réclamé un laboratoire d'expérimentation pour étudiants et jeunes professionnels. Cette approche a beaucoup de mérite pédagogique et créatif et fait appel à un certain nombre de bénévoles, mais comporte l'inconvénient de ne pas intégrer la participation citoyenne dès le processus de création. L'engagement citoyen apparaît effectivement une fois l'établissement des bases de l'organisation de l'espace et des infrastructures.

\section{2) La création d'un écosystème humain et écologique}

Par les projets éphémères, I'Université de Montréal souhaitait verdir le chantier de construction du complexe des sciences, faire de la médiation avec les collectivités limitrophes et lutter contre la résistance de l'arrivée du campus. L'objectif 


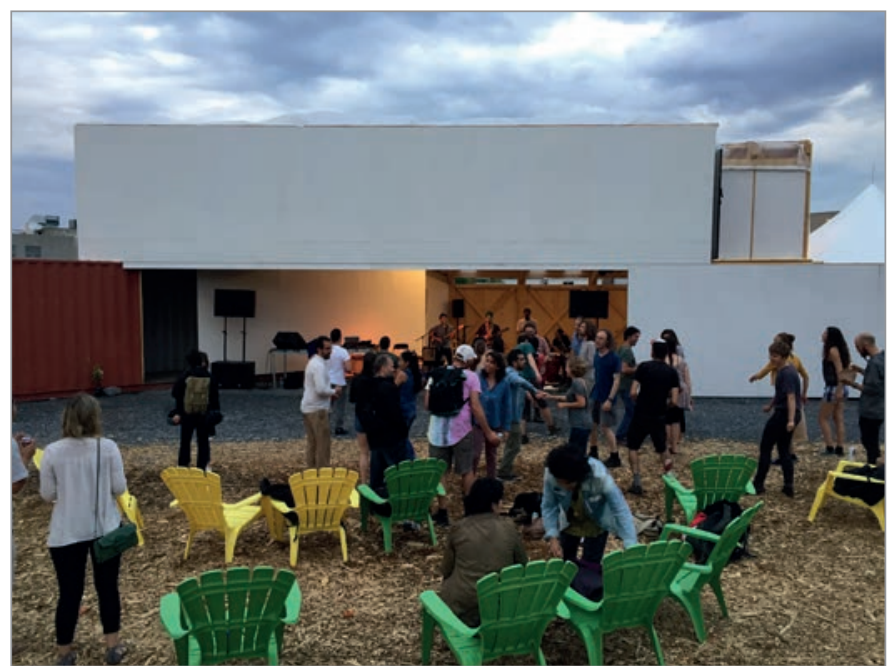

ILL. 25. PRESTATION MUSICALE DANS LE GRAND HALL ET SUR L'ESPLANADE DU VIRAGE. | JONATHAN CHA.

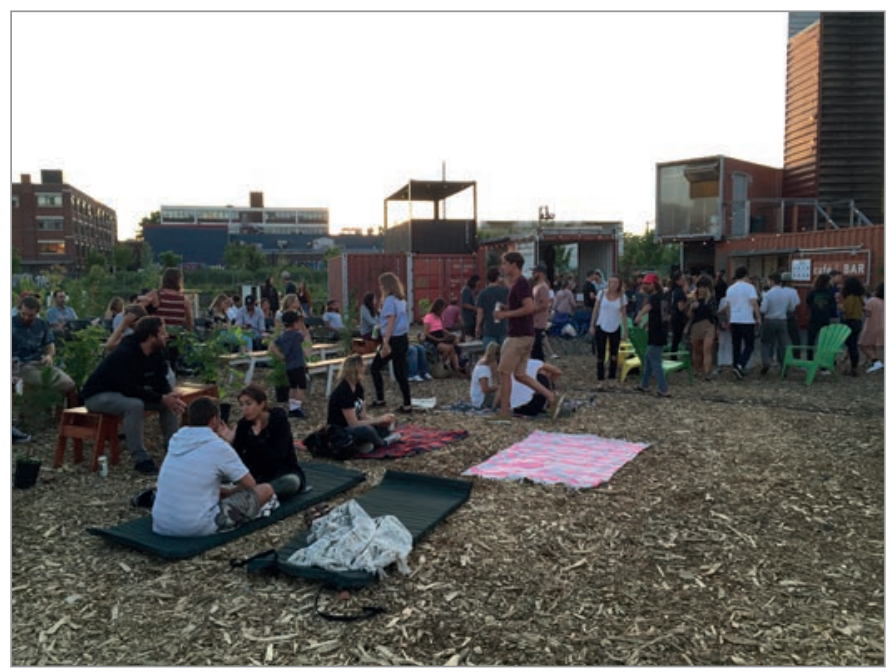

ILL. 26. OCCUPATION CITOYENNE LORS D'UNE SOIRÉE D’ÉTÉ AU VIRAGE. | JONATHAN CHA. institutionnel initial derrière ce jardin collectif en est un d'acceptabilité sociale liée aux nuisances du chantier, à l'arrivée de milliers de personnes et aux effets possibles d'embourgeoisement du quartier Parc-Extension. Ces intentions institutionnelles n'ont pas empêché les acteurs et les organismes impliqués dans la création des jardins de mettre de l'avant une pensée orientée vers les systèmes naturels, l'écologie, la biophilie et le biomimétisme.

La prise de possession de la friche industrielle a débuté par le dépôt de terre meuble sur une immense surface de gravier dans le but de créer des jardins collectifs. En 2015, cinq partenaires ont investi le lieu, onze en 2016 et vingt et un en 2017. L'agriculture urbaine, l'aménagement de la ville et le vivre ensemble sont au cœur de l'investissement du lieu. Ici on cultive, on éduque, on récolte. Un article du quotidien Le Devoir présente ce lieu émergent, ce jardin hors de l'ordinaire :

Cette surface grande comme deux terrains de football, au cœur de Montréal, est devenue un jardin collectif, un marché de légumes bio, un rucher, une pouponnière d'arbres, un cinéma en plein air et un lieu de spectacles et de réflexion sur la vie en ville. Près de 200 personnes font vivre cette place hors de l'ordinaire qui foisonne d'abeilles, d'insectes et d'une centaine de variétés de plantes. D'étranges bâtiments, formés d'une douzaine de conteneurs, ont poussé sur les lieux. Une pyramide en bois sert à la fois de gradins pour les spectacles et de point de rencontre à l'abri du soleil. Un café-bar offre boissons et collations ${ }^{57}$

La volonté des intervenants était de créer, à travers un grand potager urbain, un écosystème humain et écologique. Pour Alexandre Beaudoin, conseiller en biodiversité à l'Université de Montréal, "l'agriculture urbaine est un outil. La finalité, c'est de bâtir cet écosystème-là. Comment on le réalise, c'est à travers la mobilisation des gens pour le jardin. L'Université a créé un mécanisme pour que les gens se sentent à l'aise de s'approprier le $\operatorname{lieu}^{58}$ "). Celui-ci explique que

La première année, le terrain était inondé. Des poissons rouges ont alors été ensemencés, puis des hérons sont venus pêcher, suivis de pluviers kildir. Des ruches ont été installées, du houblon a été planté, des lapins ont été accueillis et une pépinière de 10000 arbres a pris place autour des jardins cultivables. Un réel écosystème vivant a été mis en place sur un site qui n'était que débris et amoncèlement de résidus industriels ${ }^{59}$.

Le site industriel se transforme et prend un nouveau visage vert par l'agriculture urbaine et l'engagement d'organismes communautaires qui sèment, cultivent et partagent l'espace. Le Virage et les projets éphémères permettent un dialogue direct avec le vivant, un contact et des échanges humains orientés autour de la nature cultivée et spontanée. Cet écosystème a créé un lieu de vie et d'échanges d'une rare richesse, malgré les embûches récurrentes liées au propriétaire du terrain (pas une priorité organisationnelle, pas de planification d'ensemble, pas de structure décisionnelle claire, vision à court terme, déplacement des jardins, manque de budget d'entretien et de fonctionnement) et à un objectif initial fort différent entre les intervenants. D’un côté, l'acceptabilité sociale liée à l'image de l'institution; de l'autre côté, la volonté 


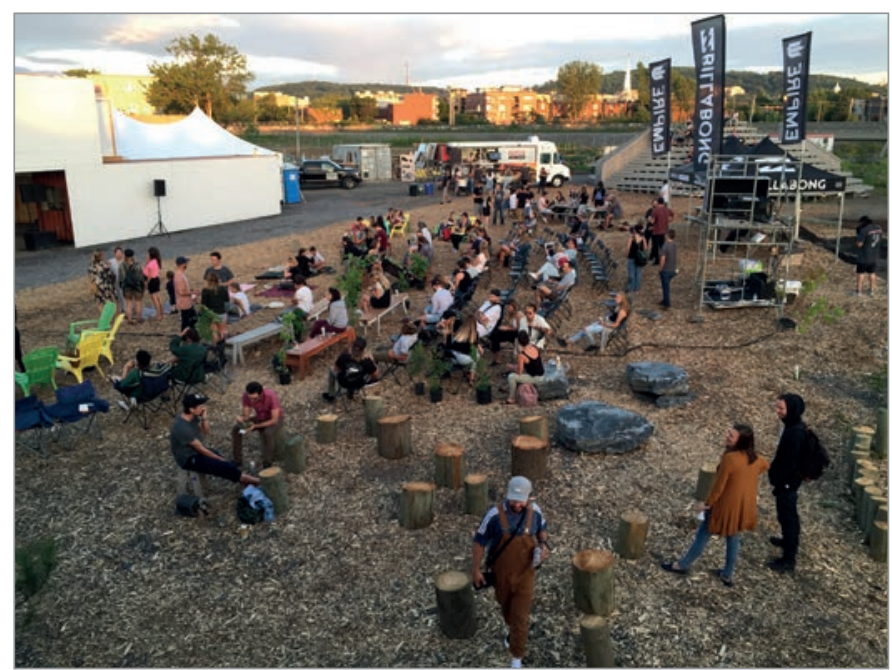

ILL. 27. UN ÉVÉNEMENT D'ÉTÉ SUR L'ESPLANADE DU VIRAGE. | JONATHAN CHA.

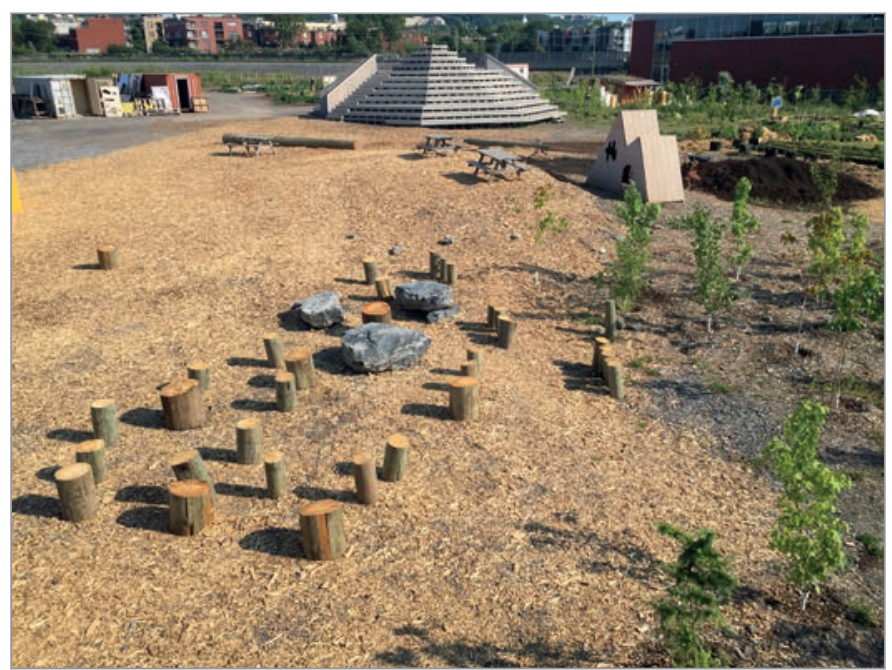

ILL. 28. DES INSTALLATIONS FLEXIBLES SUR L'ESPLANADE DU VIRAGE À PARTIR DE MATÉRIAUX RÉCUPÉRÉS. | JONATHAN CHA. partagée des organismes de créer un écosystème humain et écologique signe de changement dans la société.

\section{3) L'expérience citoyenne par la programmation culturelle, l'éducation et la formation}

Le prototypage porte en lui la volonté d'agora ${ }^{60}$, celle de créer des conditions pour l'émergence d'une urbanité, de générer une appropriation collective et du capital social. Le Virage et les projets éphémères sont un lieu d'apprentissage et de vulgarisation, "c'est le contenu qui est la véritable source d'innovation ${ }^{61} »$. Les organismes produisent du contenu qui s'adresse à des publics intéressés et variés : "formations, ateliers, cinéma en plein air, spectacles, conférences: les citoyens sont invités à plonger dans une programmation d'activités majoritairement gratuites qui allie culture, agriculture, science et citoyenneté ${ }^{2}$ " . L'organisme Miel Montréal offre des ateliers sur la biologie de l'abeille, la pollinisation, la gestion d'un rucher, les plantes mellifères et la biodiversité en milieu urbain. Des ateliers portant aussi sur l'agriculture urbaine, la permaculture, le biomimétisme et les insectes sont accompagnés de conférences, d'un marché public et de séances de cinéma en plein air. Des journées thématiques sur I'astronomie ou la Fête des récoltes accueillent des publics considérables. MVM a organisé des séries de conférences sur des enjeux urbains contemporains (le verdissement de la ville, les aménagements résilients, le design urbain écologique, la vision d'avenir des écoles, les plateformes d'innovations sociales) ${ }^{63}$, des installations artistiques, des prestations musicales, des projections en présence des cinéastes et des journées de formation professionnelle comme celle portant sur la gestion des eaux pluviales et des phytotechnologies ${ }^{64}$. Tous les publics de tous âges sont invités à venir apprendre, discuter, débattre sur ce site éducatif, rassembleur et utile, même si cet objectif demeure difficile à atteindre, notamment en termes d'inclusion sociale et de diversité culturelle. Selon le quotidien Le Devoir, c'est « un lieu de rendezvous. De partage. De rassemblement. C'est une sorte d'expérience sociale : les résidents se mobilisent pour améliorer leur quartier si les institutions leur en donnent la possibilité ${ }^{5}$. " Pour Beaudoin, "On a la responsabilité de repenser nos villes, la gestion de l'eau pluviale et les îlots de chaleur, par exemple. Par cette programmation offerte aux citoyens, on leur tend la main en souhaitant qu'ils se réapproprient l'espace, mais aussi leur ville ${ }^{66}$." "Il y a de l'amour qui se dégage du site. Les gens s'approprient le lieu ${ }^{67}$ ", précise-t-il. Les citoyens peuvent se questionner, apprendre, comprendre pour pouvoir ensuite agir. Ces nouveaux espaces communs donnent accès à des équipements culturels, contribuent au sentiment d'appartenance et d'inclusion. C'est ainsi que l'espace public peut "prendre place" et que s'activent de nouvelles dynamiques relationnelles ${ }^{68}$. L'appropriation du projet par les citoyens ne se fait pas seule et le contexte de ville festive oblige, c'est par l'animation culturelle et événementielle que s'amorce le développement d'un lien social et que $s^{\prime}$ amplifient ses usages ${ }^{69}$. Mais ici, la transmission du contenu passe par une programmation éducative, voire scientifique, par de petits auditoires dans des espaces restreints et par les échanges plutôt que 


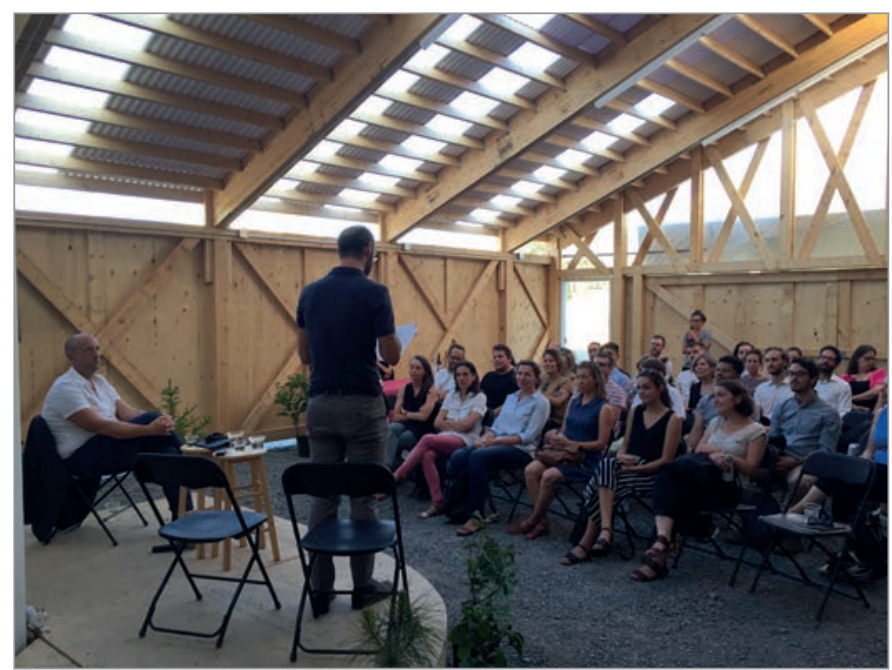

ILL. 29. UNE CONFÉRENCE DANS LE GRAND HALL AU VIRAGE. | JONATHAN CHA.

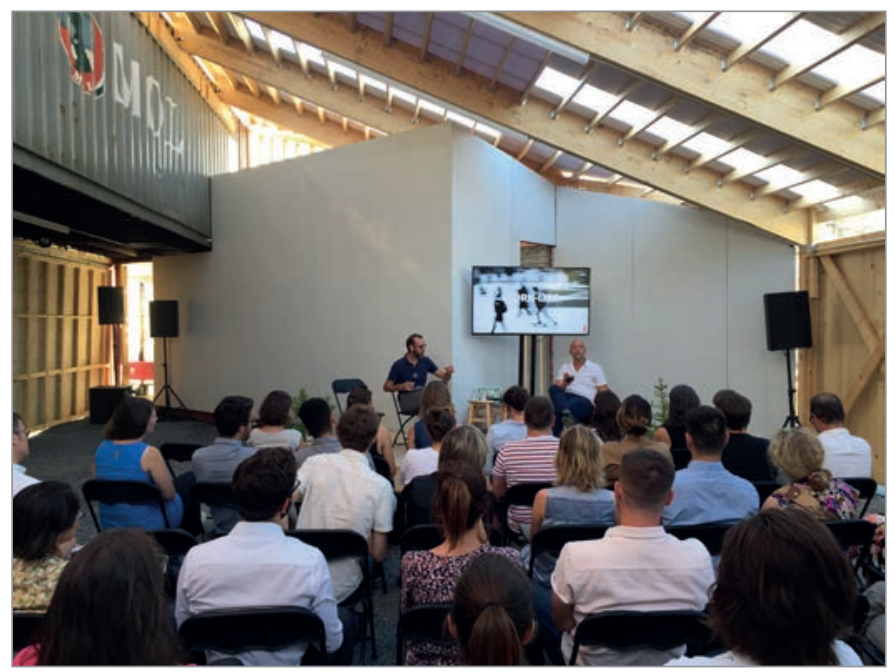

ILL. 30. UNE CONFÉRENCE DANS LE GRAND HALL AU VIRAGE. | JONATHAN CHA. par la consommation générique ou le spectacle à grand déploiement où les gens se tiennent à distance socialement. En venant sur le site du Virage et des projets éphémères, on vit une expérience d'espace public inédite par l'acquisition de connaissances, le contact avec les autres et l'action sur le site. Cette approche permet une participation accrue, mène à un engagement citoyen puis à un sentiment d'appartenance. Une telle programmation nécessite toutefois de grandes ressources humaines et financières en termes de conception artistique, de production de contenu, de coordination et de communications, pour aller rejoindre les citoyens de tous horizons, sans parler des équipements qui peuvent être utiles pour des activités à moyen ou grand déploiement (écrans, projecteurs, systèmes de son, lumières, etc.). Il faut beaucoup d'énergie et d'investissements pour livrer et engager cette expérience citoyenne si cruciale au prototype du Virage. Malgré le succès de son programme, le nombre de participants est souvent demeuré faible, en deçà des attentes par rapport à la qualité de l'expérience proposée. Ceux présents $y$ étaient toutefois fortement engagés.
4) L’adaptation, l'agilité et la flexibilité

La clé d'un prototype est l'adaptation ${ }^{70}$ et le caractère évolutif du projet ${ }^{71}$. Il régule et interconnecte les dynamiques et les couches. Le Virage et les projets éphémères agissent dans un contexte atypique non régi par des structures claires et des mécanismes éprouvés. Cela requiert donc de la souplesse, tant dans la gestion que dans le fonctionnement. MVM a expérimenté des mécanismes d'occupation, de médiation, de construction et de réflexion afin d'introduire la notion d'espace public dans ce non-lieu ${ }^{72}$. Le projet «Pla Buits» de Barcelone est inspirant par la «remise en cause des règles urbanistiques adaptées aux usages pensés comme définitifs au profit d'une flexibilisation en faveur des usages provisoires des espaces libres ${ }^{73}$ " . Dans cette idée de la ville malléable, I'importance du temps, la polyvalence de l'espace et des usages alternés prévalent ${ }^{74}$. La Virage incarne la nature provisoire et exploratoire du prototypage et implique des essais, des erreurs et des insuccès. «Pour innover, il faut se laisser une marge de manœuvre, un droit à l'erreur ${ }^{75}$. " Le Virage a activé un espace en créant ce que
I'association Cabanon Vertical nommerait " un temps d'échange et de dialogue ${ }^{76}$ ». Son occupation a fluctué lors des trois premières années : en 2016, le Virage a été actif quatre jours, en 2017, quinze jeudis soir et, en 2018, quarante-cinq jours et soirs. La transformation de cette friche a produit de l'espace public sans recourir à des typologies héritées de la ville classique, qui auraient été synonymes de réglementation, d'ordonnancement et de coûts onéreux. Cette nouvelle forme d'espace public se traduit par un citoyen participant et une succession de projets éphémères et d'occupations créatives. Le Virage se définit comme une expérimentation terrain qui révèle des solutions ${ }^{77}$; il est un moyen d'engager les parties prenantes et de stimuler le débat ${ }^{78}$. II explore les limites et les possibilités de communication et de collaboration. Il doit être à l'écoute et s'adapter aux besoins des citoyens, ce qui nécessite de l'ouverture et du temps pour gérer les éléments qui composent et recomposent l'espace. Le ménagement du site propose plusieurs espaces de sociabilité qui interagissent avec les jardins des projets éphémères et le paysage environnant. Les activités du Virage et des projets éphémères 


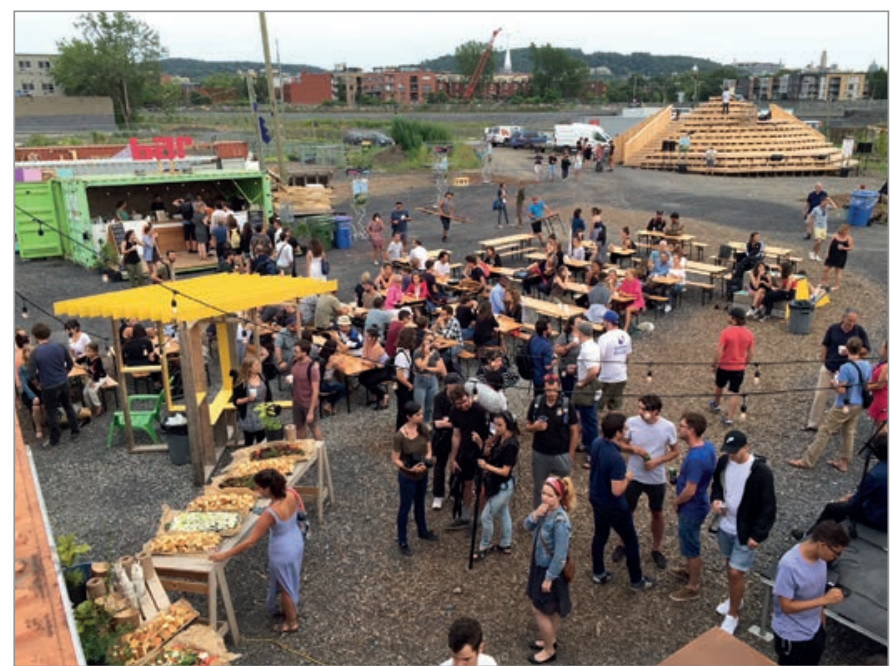

ILL. 31. UN ÉVÉNEMENT GASTRONOMIQUE AU VIRAGE; À L'ARRIÈRE-PLAN, UNE VUE SUR L'INSTALLATION DU « MONT RÉEL ». | JONATHAN CHA.

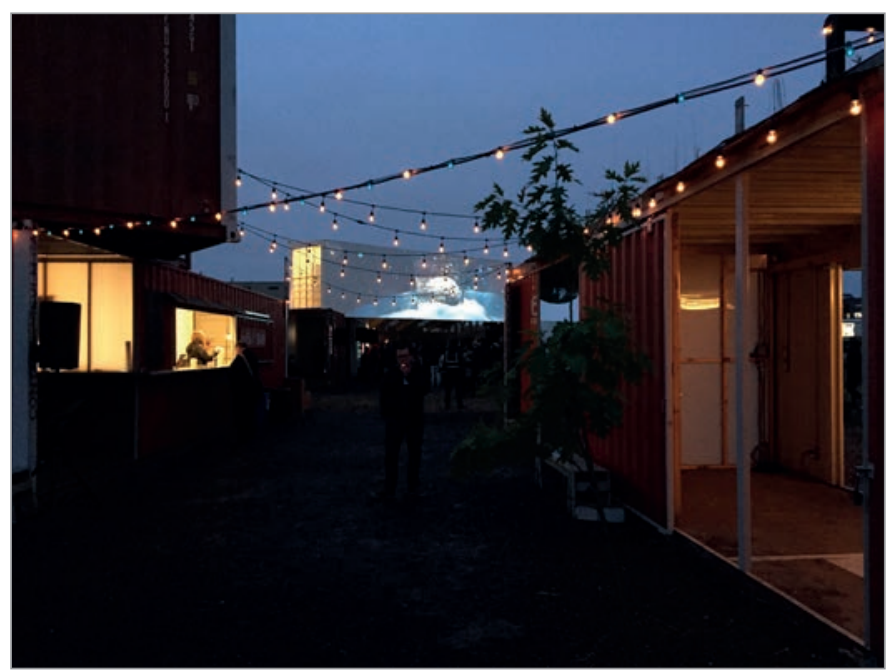

ILL. 32. LE VIRAGE LA NUIT AVEC UNE PROJECTION DE «MOMENT FACTORY», UN STUDIO MULTIMÉDIA SPÉCIALISÉ DANS LA CONCEPTION ET LA PRODUCTION D'ENVIRONNEMENTS IMMERSIFS. | JONATHAN CHA. «mettent la participation citoyenne au cœur de la démarche en donnant la possibilité d'acquérir des outils pour devenir un acteur de changement et permettent d'aborder la question environnementale d'une façon éducative ${ }^{79}$ ». Le lieu est abordable et adaptable. Comme l'écrit dans le Chicago Reader Maya Dukmasora ${ }^{80}$, qui considère les jardins communautaires comme transformateurs de la société urbaine, les villes doivent adopter une posture de spontanéité et offrir des espaces pour s'exprimer, expérimenter, tester, conceptualiser. L'évolution de ces nouvelles pratiques agiles et citoyennes ${ }^{81}$ permet d'influencer la manière de façonner l'espace public et de répondre au « désir de ville ». L'adaptation, I'agilité, la flexibilité et l'informalité constituent des ingrédients importants des formes émergentes d'espaces publics.

\section{5) La liberté d'action citoyenne}

Le Virage est un espace en mouvement où le sol, la végétation, le mobilier et l'architecture évoluent au gré des installations et des occupations. Tout n'est pas imposé, mais testé, demande à être validé avec les citoyens. L'architecture est malléable (elle s'ouvre, se ferme, se prolonge), l'espace est faiblement aménagé et laisse la liberté aux gens de le transformer, de se l'approprier à leur guise (notamment par le déplacement du mobilier, l'usage varié des espaces ou des installations temporaires). Il rapproche plutôt qu'il met à distance. Comme le souligne Delbaere, c'est "l'articulation du social sur le spatial qui fait I'espace public ${ }^{82}$. Ce prototype a un degré d'indétermination et de spontanéité pour que les citoyens imaginent leur espace collectif désiré ${ }^{83}$. Ce type de projet vise l'intérêt général, il intègre un processus d'adhésion, un engagement des gens dans le processus d'occupation. Il s'accompagne donc d'une dose d'humilité en laissant place aux agents de changement qui développent un sentiment d'appartenance au lieu. La participation citoyenne est au cœur de l'expérimentation. Ce sont les usagers qui occupent, modifient, plantent et interagissent avec I'espace. Leurs actions, leurs volontés et leur créativité deviennent une contribution (input) pour générer des idées sur le futur du lieu. C'est là que réside ce que des auteurs nomment le caractère alternatif ${ }^{84}$ ou le modèle urbain alternatif ${ }^{85}$. Selon Orduña-Giró et Sébastien Jacquot, la participation serait une solution pour l'aménagement urbain en temps de crise ${ }^{86}$. Le Virage «vient contester la logique de stricte imperméabilité qui caractérise d'ordinaire les chantiers de construction ${ }^{87}$ " en offrant des potentiels de contacts informels dans un paysage changeant au gré de la programmation et des occupations.

Au même titre que NDSM Werf ${ }^{88} \mathrm{~d}^{\prime} \mathrm{Ams}$ terdam, l'espace libre d'action est un ingrédient clé qui définit le Virage. "NDSM has a way of being, of breathing, that no other place in the city has ${ }^{89}$." Sa directrice, Kim Tuin, croit que "the site's character as a free space must be retained ${ }^{90}$ ». Cet espace hérité à contre-courant des pratiques urbanistiques accueille des pionniers urbains engagés à créer des espaces démocratiques au bénéfice de tous. Ces lieux de liberté répondent au questionnement de Benjamin Pradel qui s'interroge sur la capacité qu'ont aujourd'hui les espaces publics classiques de la ville à "faire société " au quotidien ${ }^{91}$. Les nouvelles formes d'espaces 


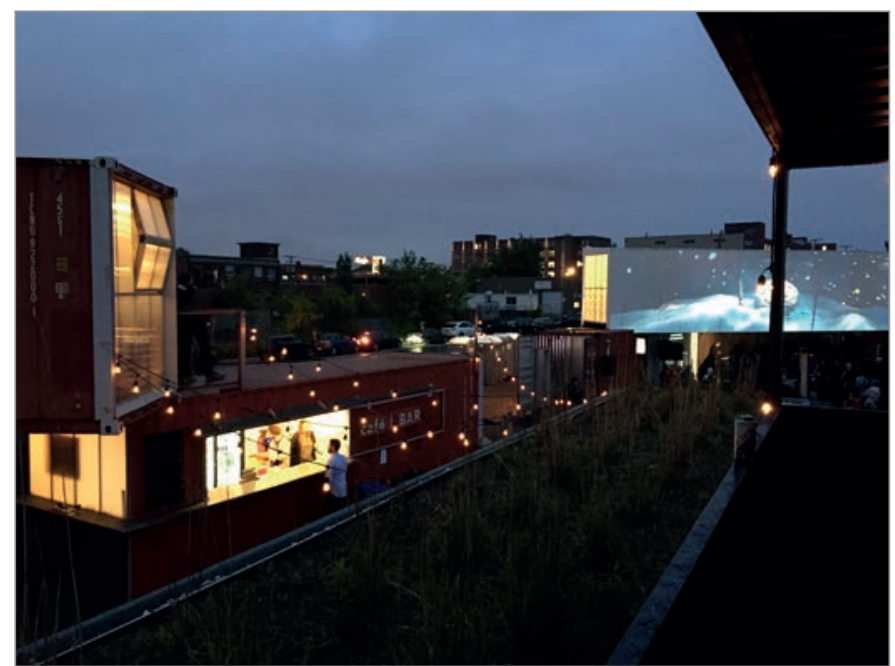

ILL. 33. LE VIRAGE LA NUIT AVEC UNE PROJECTION DE « MOMENT FACTORY». | JONATHAN CHA.

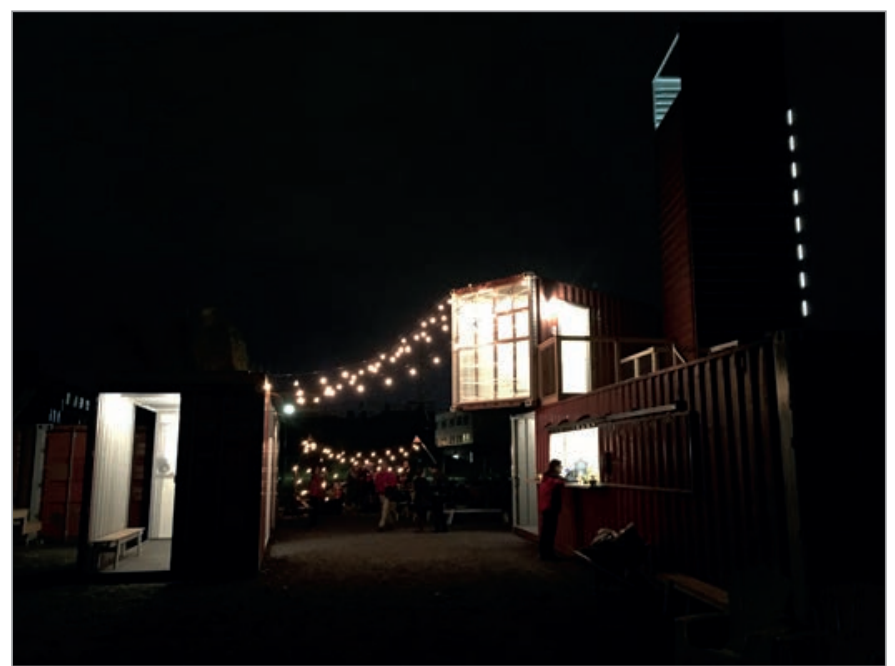

ILL. 34. LE VIRAGE LA NUIT. | JONATHAN CHA. publics tels le Virage et NDSM insufflent une dose de liberté, d'indétermination et d'incertitude ${ }^{92}$ qui attirent ceux qui délaissent les espaces publics traditionnels centraux critiqués pour leur «tendance à leur normalisation, fonctionnalisation, sécurisation et privatisation ${ }^{93}$ » ou leur «spécialisation ${ }^{94}$ ».

\section{VERS DE NOUVEAUX} ESPACES COMMUNS

L'expérience imparfaite, partiellement structurée et évolutive du Virage et des projets éphémères a mis de l'avant des principes propres aux communs qui représentent un «moyen efficace de gérer des ressources de manière équitable et pérenne, tout en renforçant les liens sociaux qui tissent des communautés résilientes et durables ${ }^{95}$ ». L'accès au territoire n'est jamais apolitique et ici c'est par le prototype de la coconstruction créative, de l'écosystème humain et écologique, de l'agriculture urbaine, de l'expérience citoyenne basée sur l'éducation, de l'agilité et de la liberté d'action que de nouveaux possibles ont pu émerger. Laure Waridel définit bien les communs:
Ni privés ni publics, les communs offrent une réponse à des problèmes de dépossession et d'exclusion. Ils remettent en question le paradigme de la propriété individuelle exclusive. Privilégiant l'usage des ressources sur leur détention, ils développent des processus collectifs [commoning] dont une communauté se dote pour gérer des ressources sur lesquelles elle revendique des droits. Les communs favorisent la création de richesse par la mise en commun de ressources intellectuelles, sociales, matérielles et environnementales ${ }^{96}$

Il s'agit d'un nouveau paradigme qui considère davantage la valeur d'usage des espaces, qui s'inscrit dans la temporalité et la pérennité et qui correspond à une gestion collective de ressources mises en partage par une communauté d'usagers. Cette troisième forme de propriété qui se distingue des propriétés publiques et privées naît d'une réappropriation citoyenne d'espaces appréhendés comme des ressources et des territoires à mieux partager, occuper et gérer. Bien que la démarche du Virage et des projets éphémères ne réponde qu'à une portion de la définition complexe des communs, il y a certes un engagement pour la collectivité qui transcende toutes les actions menées par les partenaires. En s'inspirant des villes de Détroit et de Cleveland notamment, il y a là une occasion d'expérimenter de nouvelles stratégies et politiques dans la gestion des espaces urbains et des terrains inutilisés pour leur donner de nouveaux sens et usages afin de répondre aux enjeux de la ville contemporaine ${ }^{97}$. Il est dès lors pertinent d'ajouter une couche de syntaxe urbaine, d'optimiser ces lieux indéterminés parsemés dans la structure urbaine ou aux confins des zones formalisées, de faire de ces "foyers urbains" une connectivité urbaine comme nouveau paradigme postfonctionnaliste ${ }^{98}$. L'expérience transformative vécue par les usagers durant le processus d'interaction avec le Virage et les projets éphémères permet de développer un sentiment d'appartenance visà-vis le lieu et contribue à une prise de conscience individuelle et collective de la capacité d'action des citoyens sur leur environnement. Telle est l'expérience citoyenne, celle qui engage le dialogue avec l'espace. 
LE VIRAGE, UN PREMIER PAS VERS LA RÉINVENTION DES ESPACES PUBLICS

Les projets éphémères sont nés d'une démarche institutionnelle d'acceptabilité sociale d'un grand projet immobilier perçu négativement par ses populations limitrophes. Les organismes invités à investir le lieu structuré autour du Virage, dont particulièrement MVM, ont misé sur la coconstruction créative, l'écosystème humain et écologique, l'agriculture urbaine, l'expérience citoyenne basée sur l'éducation, l'agilité et la liberté d'action pour développer une nouvelle forme d'espace public indéterminable, inclusive et évolutive.

L'expérience du Virage a pris fin abruptement, au bout de trois ans. La notoriété et la popularité d'autres lieux éphémères, la position excentrée, invisible dans la ville, et la difficulté à générer des revenus autonomes et à attirer des gens n'ont pas permis d'expérimenter pleinement le lieu et le processus ni d'atteindre des objectifs tels que l'équité sociale et la justice environnementale. En 2019 et 2020, les jardins éphémères ont continué d'exister, mais le Virage est resté inutilisé et fermé en raison du manque d'appui de I'Université de Montréal, dont un litige juridique sur la notion de propriété qui a été un frein à l'obtention d'un financement adéquat et au développement de contenus et de partenariats. L'Université de Montréal a laissé tomber MVM et Le Virage malgré le succès de sa démarche et a mis fin à l'expérimentation de la fabrique des espaces publics. La convoitise du terrain et les pressions immobilières freinent l'Université dans la cession à moyen terme du terrain à des organismes pour maintenir des usages temporaires et une nouvelle forme émergente d'espace public à vivre et à étudier. Bien que cet "entre-lieu transitoire" soit porteur d'une approche sociale et urbaine et d'un potentiel d'être un commun ou un espace propre à la ville contemporaine, I'Université de Montréal ne semble pas encore habilitée à chapeauter un tel lieu et de tels acteurs dans une vision d'innovation sociale et de recherche-action. Pendant ce temps, tout autour de ce lieu émergent dont l'activation a partiellement cessé, un grand bâtiment institutionnel, une vaste place publique avec fontaines, des rues plantées et des tours d'habitation sont inaugurés et poursuivent les manières traditionnelles de faire la ville.

Le prototype du Virage et des projets éphémères s'inscrit en plusieurs points dans la réflexion sur les communs. Il agit sur le processus, sur l'occupation de lieux en transition, interroge notre société, la gestion de nos ressources et territoires et se questionne sur les formes d'espaces publics que nous voulons pour nos villes. Comment ce type d'initiative peut-il devenir un nouveau modèle dominant pour accompagner la rénovation d'un quartier, influencer son devenir et éviter la répétition de dynamiques de standardisation et $d^{\prime}$ 'homogénéisation ${ }^{99}$ ? Comment de telles actions peuvent-elles réaliser un transfert dans le design urbain ${ }^{100}$ ? Comment ménager et prendre soin d'un lieu sans lui donner une forme finale et en favorisant une participation citoyenne et une occupation plus libre et flexible de l'espace? Il existe néanmoins des risques liés à cette approche de la ville que plusieurs auteurs ont révélés, notamment qu'elle ne soit portée que par des groupes spécifiques $^{101}$, soit politisée ${ }^{102}$ ou encore soit institutionnalisée ou appropriée à des fins simplement commerciales ou d'embellissement urbain. À cela s'ajoutent les risques réels et bien visibles que cet urbanisme tactique pionnier et alternatif entraîne un embourgeoisement ${ }^{103}$ et «soit institué comme un moyen de poursuivre les politiques néolibérales de développement urbain ${ }^{104} »$.
Cet article a fait la démonstration qu'il existe une vision alternative de la ville et de ses espaces publics, que de nouveaux et nombreux acteurs interviennent dans le processus d'aménagement urbain, qu'il y a de la place pour l'innovation et l'expérimentation dans la ville et qu'il y a d'autres façons de créer et d'occuper l'espace urbain sous-utilisé. Grâce à toutes ces initiatives, dont le prototype du Virage a été porteur, la réflexion est ouverte à une nouvelle approche de la fabrique des espaces publics de la ville contemporaine.

\section{NOTES}

1. de Smet, Aurélie, 2013, "The Role of Temporary Use in Urban (re)Development: Examples from Brussels", Brussels Studies, General Collection, $n^{\circ}$ 72, p. 1-11.

2. Montréal est le chef de file canadien en matière d'espaces éphémères. L'organisme La Pépinière espaces collectifs, qui a créé notamment le Village Au-pied-du-courant, est l'acteur le plus engagé dans la création de milieux de vie au cœur des communautés et est porté par une volonté de rendre les villes plus participatives. Si la majorité des projets éphémères portent une esthétique ludique qui leur confèrent une similitude, certains projets initiés par des partenaires privés ou la Ville de Montréal ont des visées essentiellement lucratives ou d'animation urbaine qui contrastent avec les fondements sociaux et l'apport à la réflexion urbaine de La Pépinière espaces collectifs ou de Montréal ville en mouvement (MVM).

3. Expression d'Aurélie de Smet, "The Role of Temporary Use », op. cit.

4. Lydon, Mike et Anthony Garcia, 2015, Tactical Urbanism: Short-term Action for Longterm Change, Washington, Island Press; Davis, Brian, 2010, "On Broadway, Tactical Urbanism », faslanyc.blogspot.com, 6 juin.

5. Nédélec, Pascale, 2017, "De nouveaux mots pour de nouvelles modalités de fabrique de la ville? Initiatives citadines d'aménagement des espaces publics ", Information géographique, vol. 81, n³, p. 94-107, à la p. 97.

6. REBAR (Rebar Art and Design Studio) est un studio interdisciplinaire créé en 2004. 
7. Ibid.; et Douay, Nicolas et Maryvonne Prévot, 2016, "Circulation d'un modèle urbain 'alternatif'? Le cas de I'urbanisme tactique et de sa réception à Paris ", EchoGéo, n³6, p. 1-26, aux p. 4-5.

8. Nédélec, "De nouveaux mots pour de nouvelles modalités de fabrique de la ville?", op. cit.

9. Boutleux, Dimitri, 2015, «Faire la ville autrement grâce à l'urbanisme tactique », Place publique, mai-juin, p. 30

10. Vachon, Geneviève, Érick Rivard et Alexandre Boulianne, 2015, "La micro-intervention pour comprendre, révéler et faire l'espace public ", Inter art actuel, $\mathrm{n}^{\circ} 120$, printemps, p. 8-13, à la p. 9.

11. Harvey, David, 2014, "The Crisis of Planetary Urbanization », dans Pedro Gadanho, Richard Burdett, Teddy Cruz, David Harvey, Saskia Sassen et Neder Tehrani (dir.), Uneven Growth: Tactical Urbanisms for Expanding Megacities, New York, The Museum of Modern Art, p. 26-31, à la p. 29; et Brenner, Neil, 2015, "Is 'Tactical Urbanism' an Alternative to Neoloberal Urbanism? ", Post: Notes on Modern and Contemporary Art around the World (The Museum of Modern Art online resource), mis en ligne le 24 mars, n.p., [https://teputahi.org.nz/wp-content/ uploads/2015/08/Is-"Tactical-Urbanism"-anAlternative-to-Neoliberal-Urbanism.pdf], consulté en août 2020.

12. Vachon et al., "La micro-intervention » op. cit., p. 12.

13. Id., p. 13.

14. Douay et Prévot, "Circulation d'un modèle urbain 'alternatif'?», op. cit., p. 20.

15. Courage, Cara, 2013, "The Global Phenomenon of Tactical Urbanism as Indicator of New Forms of Citizenship ", dans Karen Raney (dir.), Engage 32: Citizenship and Belonging, Londres, Engage, National Association for Gallery Education, p. 88-97.

16. Königs, Monte, 2012, "It's Not Just a Solution, It's a Way of Life ", The Protocity.com, [http:// theprotocity.com/its-not-just-a-solution-its-away-of-life/], consulté le 8 juillet 2020

17. Haydn, Florian et Robert Temel (dir.), 2006, Temporary Urban Spaces: Concepts for the Use of City Spaces, Basel, Birkhäuser.

18. Vachon et al., "La micro-intervention » op. cit., p. 8.

19. Delbaere, Denis, 2010, La fabrique de l'espace public, Paris, Ellipses.
20. Solà-Morales, Ignasi, 1995, "Terrain Vague», dans Cynthia C. Davidson (dir.), Anyplace, Cambridge, MIT Press, p. 118-123.

21. Du terme "pauzelandschappen" tiré de Bomas, Bart et Jan de Graaf, 2005, In de Tussentijd [Pendant ce temps], Wageningen, Stichting Lijn in Landschap.

22. Whyte, William H., 1989, City: Rediscovering the Centre, New York, Doubleday, p. 75.

23. Tardiveau, Armelle et Daniel Mallo, 2014, "Unpacking and Challenging Habitus: An Approach to Temporary Urbanism as a Socially Engaged Practice ", Journal of Urban Design, vol. $19, n^{\circ} 4$, p. 456-472, à la p. 459

24. L'activation est un processus ou une opération qui donne forme et sens à un lieu par un engagement citoyen.

25. Faraone, Claudia et Andrea Sarti, 2008 "Intermittent Citites on Waiting Spaces and How to Inhabit Transforming Cities", Architectural Design, vol. 78, n 1, p. 40-45.

26. Cabanon vertical, 2017, Les aménagements urbains transitoires, Enjeux et guide pratique pour un espace public partagé, p. 10, [https:// issuu.com/cabanonvertical/docs/action transitoire cabanonvertical], consulté le 8 juillet 2020.

27. Cha, Jonathan, 2017, "Le Virage et ses abords, un entre-lieu transitoire révélateur de potentiel», Échelles, n 1, «Non-lieu», p. 22-29.

28. Turgeon, Laurier, 1998, "L'état des entrelieux», dans Laurier Turgeon (dir.) Les entrelieux de la culture, Québec, L'Harmattan et Presses de I'Université Laval, p. 17.

29. Augé, Marc, 1992, "Des lieux aux non-lieux», Non-lieux, introduction à une anthropologie de la surmodernité, Paris, Seuil, p. 100.

30. Le prototype du Virage est un lieu physique où opérer une recherche-action sur les processus de fabrication et d'occupation des espaces publics. Le système d'action, d'observation et de réflexion propre à la recherche-action est une approche complémentaire à la recherche traditionnelle.

31. En creux et à la frontière entre les quartiers et les arrondissements Outremont, Parc-Extension et La Petite-Patrie (Mile-Ex ou Marconi-Alexandra) au cœur de l'île de Montréal, Le Virage et les projets éphémères s'approprient depuis 2015 une portion de l'ancienne gare de triage désaffectée du Canadien Pacifique. Celle-ci est en cours de transformation pour accueillir un futur quartier organisé autour du nouveau campus MIL de l'Université de Montréal. À l'est du vaste territoire en régénération, jusqu'à récemment nommé "site Outremont», se situent les projets éphémères formés de jardins cultivés et d'une agora composée de conteneurs créée par l'organisme MTL ville en mouvement (MVM).

32. Tardiveau et Mallo, "Unpacking and Challenging Habitus», op. cit., p. 460.

33. Brenner, «Is 'Tactical Urbanism' an Alternative to Neoloberal Urbanism», op. cit.

34. Le projet a été financé par la Ville de Montréal et I'Université de Montréal.

35. Les principaux acteurs de MVM qui ont géré et activé Le Virage de 2016 à 2018 étaient Bruno Jobin, directeur général, Jonathan Cha, codirecteur du Virage MTL, et Laurent Gauthier, coordonnateur à la programmation du Virage MTL.

36. Lemieux, Nadia, 2018, "Une place publique pour rapprocher le public du savoir scientifique ", TVA Nouvelles, 4 juin.

37. Vachon et al., "La micro-intervention » op. cit., p. 9.

38. Oswalt, Philipp, Laus Overmeyer et Philipp Misselwitz, 2013, Urban Catalyst: The Power of Temporary Use, Berlin, DOM Publishers.

39. Leur Urban Prototyping Festival (UPF) vise d'ailleurs à solliciter, tester et déployer des projets numériques et physiques dans le tissu urbain. La firme Gehl Architects a participé au premier UPF de San Francisco en 2012. Le festival est un excellent moyen de tester de nouvelles idées, de générer un dialogue et de créer des processus itératifs rapides. C'est pourquoi MVM a créé Frontière, un événement grand public qui aborde des thèmes d'actualité (comme l'intelligence artificielle) se déroulant sur le site du Virage.

40. [https://geh/people.com/blog/urban-prototyping-exploring-temporary-permanent/], consulté le 12 février 2020.

41. Pradel, Benjamin, 2011, "L'urbanisme temporaire : signifier les "espaces-enjeux» pour réédifier la ville», dans Yves Bonny, Sylvie Ollitrault, Regis Keerle et Yvon Le Caro (dir.) Espaces de vie, espaces-enjeux : entre investissements ordinaires et mobilisations politiques, Rennes, Presses universitaires de Rennes, p. 245-257, à la p. 251.

42. On constate un engouement pour la participation citoyenne, le codesign et le prototypage depuis quelques années en Amérique du Nord et en Europe particulièrement. 
43. Mayhew, Pam J. et Peter A. Dearnley, 1987, "An Alternative Prototyping Classification ", The Computer Journal, vol. 30, n 6, p. 481-484.

44. Floyd, Christiane, 1984, "A Systematic Look at Prototyping ", dans Reinhard Budde, Karin Kuhlenkamp, Lars Mathiassen et Heinz Zullighoven (dir.), Approaches to Prototyping, Heidelberg, Springer-Verlag, p. 1-18.

45. Schwartz, Frederic J., 2013, «Prototopia: Craft, Type and Utopia in Historical Perspective", dans Louise Valentine (dir.), Prototype: Design and Craft in the $21^{\text {st }}$ Century, Londres, Bloomsbury, p. 115-124.

46. Sanders, Elizabeth B.-N. et Pieter Jan Stappers, 2014, «Editorial», CoDesign, vol. 10, n 1, p. 1-4.

47. Expression utilisée par Tom Kelley, dans O'Connor, Mary Catherine, 2012, "Urban Prototyping and the Dawn of DIY Urbanism ", 25 octobre, ZDNet.com.

48. Kimbell, Lucy et Jocelyn Bailey, 2017, "Prototyping and the New Spirit of Policymaking ", International Journal of CoCreation in Design and the Arts, vol. 13, n³, p. 214-226, à la p. 221.

49. [https://gehlpeople.com/blog/urban-prototyping-exploring-temporary-permanent/], consulté le 12 février 2020.

50. Éthier, Guillaume, 2017, «Le prototype, c'est le projet ", ARQ-Architecture et design Québec, n०180, août, "Grands projets petits projets", p. 7-9, à la p. 7.

51. Goudin-Steinmann, Elisa, 2017, «Spatialité et mise en récit de projets de vie alternatifs dans des espaces de l'ex Berlin-Est détournés de leur usage initial », Cahiers de Narratologie, $n^{\circ} 31$, p. 1-12, à la p. 6.

52. Éthier, "Le prototype, c'est le projet», op. cit., p. 8.

53. Vachon et al., "La micro-intervention » op. cit.

54. Les firmes Provencher Roy, NIPpaysage, box architectures, MU architecture et Table Architecture ont été impliquées dans le processus.

55. Rivard, Érick, 2017, "Le projet transitoire", $A R Q$-Architecture et design Québec, $\mathrm{n}^{\circ} 180$, août, "Grands projets petits projets ", p. 11-13, à la p. 13.

56. Le Virage était parmi les candidats retenus pour les Grands Prix de I'Opération patrimoine Montréal 2019, dans la catégorie Agir ensemble qui s'adresse à tout groupe (organisme à but non lucratif ou regroupement de citoyens) ayant contribué à mettre en valeur un lieu ou un secteur d'intérêt de la ville, même si l'objectif de départ n'était pas la conservation du patrimoine.

57. Fortier, Marco, 2018, "La biologie pour redonner vie à un quartier », Le Devoir, 13 juillet.

58. Ibid.

59. Ibid.

60. Ardenne, Paul, 2002, Un art contextuel, création artistique en milieu urbain, en situation d'intervention, de participation, Paris, Flammarion.

61. Éthier, "Le prototype, c'est le projet», op. cit. p. 9.

62. Université de Montréal, 2018, "L'été sera fertile en découvertes au Virage", 12 juin, [https://campusmil.umontreal.ca/2018/06/12/ lete-sera-fertile-en-decouvertes-au-viragecampus-mil/], consulté le 8 juillet 2020 .

63. Pour un résumé des conférences, voir Cha, Jonathan, 2018, "Les conférences nocturnes du Virage-Campus MIL », ARQ-Architecture et design Québec, n 185, décembre, p. 11-15.

64. Organisée par l'Association des architectes paysagistes du Québec (AAPQ), le 31 août 2018.

65. Fortier, "La biologie pour redonner vie à un quartier», op. cit.

66. Ville de Montréal, 2018, «Un rendez-vous édu catif éphémère pour une conscience durable", 3 juillet, [http://ville.montreal.qc.ca/anous/ articles/le-virage-du-campus-mil], consulté le 27 juillet 2019.

67. Fortier, «La biologie pour redonner vie à un quartier», op. cit.

68. Vachon et al., "La micro-intervention » op. cit

69. Pradel, «L'urbanisme temporaire», op. cit., p. 253.

70. Bunschoten, Raoul, 2012, "Points, Spirals and Prototypes", dans Peter Blundelle Jones, Doina Petrescu et Jeremy Hill (dir.), Architecture and Participation, Londres et New York, Routledge, p. 235-246, à la p. 246.

71. Éthier, "Le prototype, c'est le projet», op. cit.

72. Cha, "Le Virage et ses abords», op. cit.

73. Orduña-Giró, Paula et Sébastien Jacquot, 2014, "La production participative d'espaces publics temporaires en temps de crise", Métropolitiques, 7 novembre, n.p., [https:// www.metropolitiques.eu/La-productionparticipative-d.html], consulté le 8 juillet 2020.
74. Gwiazdzinski, Luc et Jérôme Massiani, 2015, Penser la ville malléable: vers une approche spatio-temporelle des politiques urbaines, Collectif d'action pour la ville/expérimentation et réflexion in situ (COLAVERI), cité dans Douay et Prévot, "Circulation d'un modèle urbain 'alternatif'?», op.cit.

75. Éthier, «Le prototype, c'est le projet», op. cit., p. 10.

76. Cabanon vertical, Les aménagements urbains transitoires, op. cit., p. 24.

77. Chow, Rosan, 2013, "The RIP + MIX Method and Reflection on its Prototypes", dans Louise Valentine (dir.), Prototype: Design and Craft in the $21^{\text {st }}$ Century, Londres, Bloomsbury, p. 155-168.

78. Franzato, Carlo, 2011, «Design as Speculation», Design Philosophy Papers, vol. 9, n 1, p. 23-39.

79. Beaudoin, Alexandre, 2018, "Jardiner à I'ombre des grues", Stratégies. Contenu. Relations médias, Exergue, Université de Montréal, 21 juin, n.p., [https://www.exergue. ca/jardiner-a-lombre-des-grues/], consulté le 8 juillet 2020.

80. Dukmasora, Maya, 2019, "Community Gardens Beautify Urban Space, but Some Seek to Transform Urban Society", Chicago Reader, 13 mars, n.p., [https://www.chicagoreader. com/chicago/community-gardens-beautifytransform/Content?oid $=68945340]$, consulté le 8 juillet 2020 .

81. Boutleux, "Faire la ville autrement», op. cit., p. 34.

82. Delbaere, La fabrique de l'espace public, op. cit., p. 79.

83. Tardiveau et Mallo, "Unpacking and Challenging Habitus», op. cit.

84. Tiré de l'appel à articles en 2013 de la revue Métropoles intitulé «Politiques alternatives de développement urbain ».

85. Douay et Prévot, "Circulation d'un modèle urbain 'alternatif'?», op. cit.

86. Orduña-Giró et Jacquot, "La production participative d'espaces publics temporaires", op. cit.

87. Éthier, Guillame, 2017, «L'urbanisme tactique comme pratique spatiale de la connectivité?", Inter, $\mathrm{n}^{\circ} 125$, p. $4-9$, à la p. 8.

88. La Nederlandsche Dok en Scheepsbouw Matschappij", était une entreprise néerlandaise de construction navale basée à Amsterdam. 
89. Tuin, Kim, 2017, "NDSM Werf to Continue as Rugged Sister to Amsterdam's Museumplein ", NDSM, 10 avril, n.p., [http://www.ndsm.nl/en/ story/ndsm-werf-to-continue-as-rugged-sister-to-amsterdams-museumplein/], consulté le 8 juillet 2020

90. Ibid.

91. Pradel, «L'urbanisme temporaire», op. cit.

92. Silva, Paulo, 2016, "Tactical Urbanism: Towards an Evolutionary Cities' Approach?», Environment and Planning B Planning and Design, vol. 43, n6, p. 1040-1051.

93. Pradel, "L'urbanisme temporaire», op. cit

94. Cha, Jonathan, 2012, "La spécialisation des places publiques au sein de quartiers thématisés du centre-ville de Montréal : Entre marquage identitaire, revitalisation urbaine et branding de ville ", dans Lyne Bernier, Mathieu Dormaels et Yann Le Fur (dir.), La patrimonialisation de l'urbain, Québec, Presses de I'Université du Québec, coll. «Les Cahiers de I'Institut du patrimoine», p. 179-205.

95. Waridel, Laure, 2018, "La pertinence des communs», Le Devoir, 20 septembre.

96. Ibid.

97. Coppola, Alessandro, 2014, «A Cleveland Model? Experiments in Alternative Urbanism in the Rustbelt », Métropoles, n 15, p. 1-16.
98. Éthier, «L'urbanisme tactique comme pratique spatiale», op. cit.

99. Harvey, David, 1989, «From Managerialism to Entrepreneurialism: The Transformation in Urban Governance in Late Capitalism ", Geografiska Annaler, vol. 71 n 1, p. 3-17.

100. Silva, "Tactical Urbanism», op. cit.

101. Wortham-Galvin, Brooke D., 2013, "An Anthropology of Urbanism: How People Make Places (and What Designers and Planners Might Learn from It) ", Footprint: Delft Architecture Theory Journal, vol. 7, n², p. 21-39.

102. Spataro, David, 2016, "Against a De-politicised DIY Urbanism: Food Not Bombs and the Struggle Over Public Space ", Journal of Urbanism: International Research on Placemaking and Urban Sustainability, vol. 9, $\mathrm{n}^{\circ} 2$, p. 185-201.

103. CoIpron, Suzanne, 2019 , «Embourgeoisement: Park-Extension sous tension ", La Presse, 30 juin.

104. Mould, Oli, 2014, "Tactical Urbanism: The New Vernacular of the Creative City", Geography Compass, vol. 8, n 8, p. 529-539. 